\title{
A general Fredholm theory I: A splicing-based differential geometry
}

Received December 20, 2006

\section{Introduction}

In a series of papers we develop a generalized Fredholm theory and demonstrate its applicability to a variety of problems including Floer theory, Gromov-Witten theory, contact homology, and symplectic field theory, [2]. Here are some of the basic common features:

- The moduli spaces are solutions of elliptic PDE's showing serious noncompactness phenomena having well-known names like bubbling-off, stretching the neck, blowup, breaking of trajectories. These drastic names are a manifestation of the fact that one is confronted with analytical limiting phenomena where the classical analytical descriptions break down.

- When the moduli spaces are not compact, they admit nontrivial compactifications like the Gromov compactification [4] of the space of pseudoholomorphic curves in Gromov-Witten theory or the compactification of the moduli spaces in symplectic field theory (SFT) as described in [1].

- In many problems like in Floer theory, contact homology or symplectic field theory the algebraic structures of interest are precisely those created by the "violent analytical behavior" and its "taming" by suitable compactifications. In fact, the algebra is created by the complicated interactions of many different moduli spaces.

In the abstract theory we shall introduce a new class of spaces called polyfolds which in applications are the ambient spaces of the compactified moduli spaces. We introduce bundles $p: Y \rightarrow X$ over polyfolds which, as well as the underlying polyfolds, can have varying dimensions. We define the notion of a Fredholm section $\eta$ of the bundle $p$ whose zero sets $\eta^{-1}(0) \subset X$ are in our applications precisely the compactified moduli spaces one is interested in. The normal "Fredholm package" will be constructed consisting of an abstract perturbation and transversality theory. In the case of transversality the solution spaces are smooth manifolds, smooth orbifolds, or smooth weighted branched manifolds (in the sense of McDuff, [16]), depending on the generality of the situation.

H. Hofer: Courant Institute of Mathematical Sciences, New York University, 251 Mercer St., New York, NY 10012-11855, USA; e-mail: hofer@cims.nyu.edu

K. Wysocki: Mathematics Department, Penn State University, University Park, State College, PA 16802, USA; e-mail: wysocki@ math.psu.edu

E. Zehnder: Departement Mathematik, ETH Zürich, Rämisstr. 1001, 8092 Zürich, Switzerland; e-mail: eduard.zehnder@math.ethz.ch 
The usefulness of this theory will be illustrated by our 'Application' series. The applications include Gromov-Witten theory, Floer theory and SFT (see [11, 12]). It is, however, clear that the theory applies to many more nonlinear problems showing a lack of compactness.

The current paper is the first in the 'Theory' series and deals with a generalization of differential geometry which is based on new local models. These local models are open sets in splicing cores. Splicing cores are smooth spaces with tangent spaces having in general locally varying dimension. These spaces are associated to splicings, which is the basic concept in this paper. The resulting local models for a new kind of smooth spaces are needed to deal with the functional-analytic descriptions of situations in which serious compactness problems arise. We would also like to note that the applications of the concepts in this paper can be viewed as a generalization of [3] to a situation where we have varying domains and targets.

The second paper, [8], develops the implicit function theorems in this general context and extends the usual Fredholm theory.

The third paper, [9], develops the Fredholm theory in polyfolds, which could be viewed as a theory of Fredholm functors in a version of Lie groupoids with object and morphism spaces build on the new local models (see [17], [18] for the groupoid concepts in a manifold world). The Fredholm theory in this generalization is sufficient to deal with the problems mentioned above.

On purpose we have not included any applications in this series since we did not want to dilute the ideas. The conceptual framework should apply to many more situations. We refer the reader to [6, 7, 11, 12] for applications on different depth levels. An overview is given in [5].

\section{Sc-calculus in Banach spaces}

In order to develop the generalized nonlinear Fredholm theory needed for the symplectic field theory, we start with calculus issues. In a first step we equip Banach spaces with the structure of a scale, called sc-structure. Scales are a well-known concept from interpolation theory (see for example [19]). We give a new interpretation of a scale as a generalization of a smooth structure. Then we introduce the appropriate class of smooth maps. Having developed the notion of an sc-smooth structure on an open subset of a Banach space as well as that of a smooth map, the validity of the chain rule allows then, in principle, to develop an "sc-differential geometry" by simply imitating the classical constructions. However, new objects are possible, with the most important one being that of a general splicing. The main purpose of this paper is to introduce them and to show how they define local models for a new class of smooth spaces, which are crucial for the aforementioned applications.

\subsection{Sc-structures}

We begin by introducing the notion of an sc-smooth structure on a Banach space and on its open subsets. 
Definition 2.1. Let $E$ be a Banach space. An sc-structure on $E$ is given by a nested sequence

$$
E=E_{0} \supseteq E_{1} \supseteq \cdots \supseteq \bigcap_{m \geq 0} E_{m}=: E_{\infty}
$$

of Banach spaces $E_{m}, m \in \mathbb{N}=\{0,1,2, \ldots\}$, having the following properties.

- If $m<n$, the inclusion $E_{n} \hookrightarrow E_{m}$ is a compact operator.

- The vector space $E_{\infty}$ is dense in $E_{m}$ for every $m \geq 0$.

In the following we shall sometimes talk about an sc-smooth structure on a Banach space rather than an sc-structure to emphasize the smoothness aspect. From the definition of an sc-structure it follows, in particular, that $E_{n} \subseteq E_{m}$ is dense if $m<n$ and the embedding is continuous. We note that $E_{\infty}$ has the structure of a Fréchet space. In the case where $\operatorname{dim}(E)<\infty$ the only possible sc-structure is the constant structure with $E_{m}=E$.

If $U \subset E$ is an open subset we define the induced sc-smooth structure on $U$ to be the nested sequence $U_{m}=U \cap E_{m}$. Given an sc-smooth structure on $U$ we observe that $U_{m}$ inherits the sc-smooth structure defined by $\left(U_{m}\right)_{k}=U_{m+k}$. We will write $E^{m}$ to emphasize that we are dealing with the Banach space $E_{m}$ equipped with the sc-structure $\left(E^{m}\right)_{k}:=E_{m+k}$ for all $k \geq 0$. Similarly we will distinguish between $U_{m}$ and $U^{m}$.

Remark 2.2. The compactness requirement is crucial for applications. It is possible to develop a theory without this requirement, but it is not applicable to the theories we are interested in. This alternative theory would in the case of the constant sequence $E_{m}=E$ recover the standard smooth structure on $E$. However, the notion of a smooth map would be more restrictive. Both theories, the one described in this paper and the one just alluded to, intersect therefore only in the standard finite-dimensional theory. See Remark 2.17 for further details.

If $E$ and $F$ are equipped with sc-structures, the Banach space $E \oplus F$ carries the scstructure defined by $(E \oplus F)_{m}=E_{m} \oplus F_{m}$.

Definition 2.3. Let $U$ and $V$ be open subsets of sc-smooth Banach spaces. A continuous map $\varphi: U \rightarrow V$ is said to be of class $\mathrm{sc}^{0}$ or simply $\mathrm{sc}^{0}$ if $\varphi\left(U_{m}\right) \subset V_{m}$ and the induced maps $\varphi: U_{m} \rightarrow V_{m}$ are all continuous.

Next we define the tangent bundle.

Definition 2.4. Let $U$ be an open subset in an sc-smooth Banach space $E$ equipped with the induced sc-structure. Then the tangent bundle $T U$ of $U$ is defined by $T U=U^{1} \oplus E$. Hence the induced sc-smooth structure is defined by the nested sequence

$$
(T U)_{m}=U_{m+1} \oplus E_{m}
$$

together with the $s c^{0}$-projection

$$
p: T U \rightarrow U^{1} .
$$

Note that the tangent bundle is not defined on $U$ but merely on the smaller, but dense, subset $U_{1}$. We shall refer to the points in $U_{m}$ sometimes as points in $U$ on level $m$. 


\subsection{Linear sc-theory}

We begin by developing some of the linear theory needed in the sc-calculus.

Definition 2.5. Consider E equipped with an sc-smooth structure.

- An sc-subspace $F$ of $E$ consists of a closed linear subspace $F \subseteq E$ so that $F_{m}=$ $F \cap E_{m}$ defines an sc-structure for $F$.

- An sc-subspace $F$ of $E$ splits if there exists another sc-subspace $G$ so that on every level we have the topological direct sum

$$
E_{m}=F_{m} \oplus G_{m} .
$$

We shall use the notation $E=F \oplus_{\mathrm{sc}} G$ or $E=F \oplus G$ if there is no possibility of confusion.

Next we introduce the relevant linear operators in the sc-context.

Definition 2.6. Let $E$ and $F$ be sc-smooth Banach spaces.

- An sc-operator $T: E \rightarrow F$ is a bounded linear operator which in addition is $s c^{0}$, i.e. it induces bounded linear operators $T: E_{m} \rightarrow F_{m}$ on all levels.

- An sc-isomorphism is a bijective sc-operator $T: E \rightarrow F$ such that $T^{-1}: F \rightarrow E$ is also an sc-operator.

An interesting class of sc-operators is the class of sc-projections, i.e. sc-operators $P$ with $P^{2}=P$.

Proposition 2.7. Let $E$ be an sc-smooth Banach space and $K$ a finite-dimensional subspace of $E_{\infty}$. Then $K$ splits the sc-space $E$.

Note that a finite-dimensional subspace $K$ of $E$ which splits the sc-smooth space $E$ is necessarily a subspace of $E_{\infty}$.

Proof. Take a basis $e_{1}, \ldots, e_{n}$ for $K$ and fix the associated dual basis. By Hahn-Banach this dual basis can be extended to continuous linear functionals $\lambda_{1}, \ldots, \lambda_{n}$ on $E$. Now $P(h)=\sum_{i=1}^{n} \lambda_{i}(h) e_{i}$ defines a continuous projection on $E$ with image in $K \subset E_{\infty}$. Hence $P$ induces continuous maps $E_{m} \rightarrow E_{m}$. Therefore $P$ is an sc-projection. Define $Y_{m}=(\mathrm{Id}-P)\left(E_{m}\right)$. Setting $Y=Y_{0}$ we have $E=K \oplus Y$. By construction, $Y_{m} \subset E_{m} \cap Y_{0}$. An element $x \in E_{m} \cap Y_{0}$ has the form $x=e-P(e)$ with $e \in E_{0}$. Since $P(e) \in E_{\infty}$ we see that $e \in E_{m}$, implying $Y_{m}=E_{m} \cap Y_{0}$. Finally, $Y_{\infty}=\bigcap_{m \geq 0} Y_{m}$ is dense in $Y_{m}$ for every $m \geq 0$. Indeed, if $x \in Y_{m}$, we can choose $x_{k} \in E_{\infty}$ satisfying $x_{k} \rightarrow x$ in $E_{m}$. Then $(\mathrm{Id}-P) x_{k} \in Y_{\infty}$ and $(\mathrm{Id}-P) x_{k} \rightarrow(\operatorname{Id}-P) x=x$ in $Y_{m}$.

We can introduce the notion of a linear Fredholm operator in the sc-setting.

Definition 2.8. Let $E$ and $F$ be sc-smooth Banach spaces. An sc-operator $T: E \rightarrow F$ is called Fredholm provided there exist sc-splittings $E=K \oplus_{\mathrm{sc}} X$ and $F=Y \oplus_{\mathrm{sc}} C$ having the following properties.

- $K=\operatorname{ker}(T)$ is finite-dimensional. 
- $C$ is finite-dimensional.

- $Y=T(X)$ and $T: X \rightarrow Y$ defines a linear sc-isomorphism.

The above definition implies that $T\left(X_{m}\right)=Y_{m}$, the kernel of $T: E_{m} \rightarrow F_{m}$ is equal to $K$, and $C$ spans its cokernel, so that

$$
E_{m}=K \oplus X_{m} \quad \text { and } \quad F_{m}=C \oplus T\left(E_{m}\right)
$$

for all $m \geq 0$. It is an easily established fact that the composition of two sc-Fredholm operators $T$ and $S$ is sc-Fredholm. From the index additivity of classical Fredholm operators we obtain the same in our set-up,

$$
i(T S)=i(T)+i(S) .
$$

The following observation, called the regularizing property, should look familiar.

Proposition 2.9. Assume $T: E \rightarrow F$ is sc-Fredholm and $T(e) \in F_{m}$ for some $e \in E_{0}$. Then $e \in E_{m}$.

Proof. Since $F_{m}=T\left(E_{m}\right) \oplus C$, the element $f=T(e) \in F_{m}$ has the representation

$$
f=T(x)+c
$$

for some $x \in X_{m}$ and $c \in C$. Similarly, $e$ has the representation

$$
e=k+x_{0}
$$

with $k \in K=\operatorname{ker}(T)$ and $x_{0} \in X_{0}$ because $E_{0}=K \oplus X_{0}$. From $T(e)=f=T(x)+c$ and $T(x)=T\left(x_{0}\right)$ one concludes $T\left(x_{0}-x\right)=c$. Hence $c=0$ because $T\left(E_{0}\right) \cap C=\{0\}$. Consequently, $x_{0}-x \in K$. Since $e-x=k+\left(x_{0}-x\right) \in K$ and $x \in E_{m}$ and $K \subset E_{m}$, one concludes $e \in E_{m}$ as claimed.

We end this subsection with the important definition of an $\mathrm{sc}^{+}$-operator and a stability result for Fredholm maps.

Definition 2.10. Let $E$ and $F$ be sc-Banach spaces. An sc-operator $R: E \rightarrow F$ is said to be an $\mathrm{sc}^{+}$-operator if $R\left(E_{m}\right) \subset F_{m+1}$ for every $m \geq 0$ and if $R$ induces an sc ${ }^{0}$-operator $E \rightarrow F^{1}$.

Let us note that due to the (level-wise) compact embedding $F^{1} \rightarrow F$ an sc ${ }^{+}$-operator induces a compact operator on every level. This follows immediately from the factorization

$$
R: E \rightarrow F^{1} \rightarrow F .
$$

The stability result is the following statement.

Proposition 2.11. Let $E$ and $F$ be sc-Banach spaces. If $T: E \rightarrow F$ is an sc-Fredholm operator and $R: E \rightarrow F$ an sc ${ }^{+}$-operator, then $T+R$ is also an sc-Fredholm operator. 
Proof. Since $R: E_{m} \rightarrow F_{m}$ is compact for every level, we see that $T+R: E_{m} \rightarrow F_{m}$ is Fredholm for every $m$. Let $K_{m}$ be the kernel of $T+R: E_{m} \rightarrow F_{m}$. We claim that $K_{m}=K_{m+1}$ for every $m \geq 0$. Clearly, $K_{m+1} \subseteq K_{m}$. To see that $K_{m} \subseteq K_{m+1}$, take $x \in K_{m}$. Then $T x=-R x \in F_{m+1}$ and, in view of Proposition 2.9. $x \in E_{m+1}$. Hence $x \in K_{m+1}$, implying $K_{m} \subseteq K_{m+1}$. Set $K=K_{0}$. By Proposition 2.7 $K$ splits the scspace $E$ since it is a finite dimensional subset of $E_{\infty}$. Hence we have the sc-splitting $E=K \oplus X$ for a suitable sc-subspace $X$. Next define $Y_{m}=(T+R)\left(E_{m}\right)$. This defines an sc-structure on $Y=Y_{0}$. Let us show that $F$ induces an sc-structure on $Y$ and that this is the one given by $Y_{m}$. For this it suffices to show that

$$
Y \cap F_{m}=Y_{m} .
$$

Clearly,

$$
Y_{m}=(T+R)\left(E_{m}\right)=F_{m} \cap(T+R)\left(E_{m}\right) \subset F_{m} \cap(T+R)\left(E_{0}\right)=Y \cap F_{m} .
$$

Next assume that $y \in Y \cap F_{m}$. Then there exists $x \in E_{0}$ with $T x+R x=y$. Since $R$ is an $\mathrm{sc}^{+}$-section it follows that $y-R x \in F_{1}$, implying that $x \in E_{1}$. Inductively we find that $x \in E_{m}$, implying that $y \in Y_{m}$, and (1) is proved. Observe that we also have

$$
F_{\infty} \cap Y=\left(\bigcap_{m \in \mathbb{N}} F_{m}\right) \cap Y=\bigcap_{m \in \mathbb{N}}\left(F_{m} \cap Y\right)=\bigcap_{m \in \mathbb{N}} Y_{m}=Y_{\infty} .
$$

In view of Lemma 2.12 below, there exists a finite-dimensional subspace $C \subset F_{\infty}$ satisfying $F_{0}=C \oplus Y$. From this it follows that $F_{m}=C \oplus Y_{m}$. Indeed, since $C \cap Y_{m} \subset$ $C \cap Y$, we have $C \cap Y_{m}=\{0\}$. If $f \in F_{m}$, then $f=c+y$ for some $c \in C$ and $y \in Y$ since $F_{m} \subset Y$ and $F_{0}=C \oplus Y$. Hence $y=f-c \in F_{m}$ and using Proposition 2.9 we conclude $y \in F_{m}$. This implies, in view of [1], that $F_{m}=C \oplus Y_{m}$. We also have $F_{\infty}=C \oplus\left(F_{\infty} \cap Y\right)=C \oplus Y_{\infty}$. It remains to show that $Y_{\infty}$ is dense in $Y_{m}$ for every $m \geq 0$. Take $y \in Y_{m}$. Then $y=(T+R)(x)$ for some $x \in E_{m}$. The space $E_{\infty}$ is dense in $E_{m}$ so that there exists a sequence $\left(x_{n}\right) \subset E_{\infty}$ converging to $x$ in $E_{m}$. The operator $T+R$ is $\mathrm{sc}^{0}$-continuous and so the sequence $y_{n}:=(T+R)\left(x_{n}\right) \in F_{m}$ converges to $y=(T+R)(x)$ in $F_{m}$. Now, in view of Proposition 2.9 and Definition 2.10, the points $y_{n}$ belong to $Y_{\infty}$ and our claim is proved. Consequently, we have the sc-splitting

$$
F=Y \oplus_{\mathrm{sc}} C
$$

and, up to Lemma 2.12 below, the proof of the proposition is complete.

Lemma 2.12. Assume $F$ is a Banach space and $F=D \oplus Y$ with $D$ of finite dimension and $Y$ a closed subspace of $F$. Assume, in addition, that $F_{\infty}$ is a dense subspace of $F$. Then there exists a finite-dimensional subspace $C \subset F_{\infty}$ such that $F=C \oplus Y$.

Proof. The quotient $F / Y$ is a finite-dimensional Banach space and we have a continuous projection operator $p: F \rightarrow F / Y$. Since $F_{\infty}$ is a dense linear subspace of $F$ we find that $p\left(F_{\infty}\right)=F / Y$. Take any basis for $F / Y$ and pick representatives for these vectors in $F_{\infty}$. Their span $C$ has the desired property. 


\subsection{Sc-smooth maps}

In this subsection we introduce the notion of an $\mathrm{sc}^{1}$-map.

Definition 2.13. Let $E$ and $F$ be sc-smooth Banach spaces and let $U \subset E$ be an open subset. An $\mathrm{sc}^{0}$-map $f: U \rightarrow F$ is said to be $\mathrm{sc}^{1}$ or of class $\mathrm{sc}^{1}$ if the following conditions hold true.

(1) For every $x \in U_{1}$ there exists a linear map $D f(x) \in \mathcal{L}\left(E_{0}, F_{0}\right)$ satisfying, for $h \in E_{1}$ with $x+h \in U_{1}$,

$$
\frac{1}{\|h\|_{1}}\|f(x+h)-f(x)-D f(x) h\|_{0} \rightarrow 0 \quad \text { as }\|h\|_{1} \rightarrow 0
$$

(2) The tangent map $T f: T U \rightarrow T F$ defined by

$$
T f(x, h)=(f(x), D f(x) h)
$$

is an $s c^{0}$-map.

The linear map $D f(x)$ will often be called the linearization of $f$ at the point $x$. If the sc-continuous map $f: U \subset E \rightarrow F$ is of class $\operatorname{sc}^{1}$, then its tangent map

$$
T f: T U \rightarrow T F
$$

is an $\mathrm{sc}^{0}$-map. If now $T f$ is of class $\mathrm{sc}^{1}$, then $f: U \rightarrow F$ is called of class $\mathrm{sc}^{2}$.

Proceeding inductively, the map $f: U \rightarrow F$ is called of class $s c^{k}$ if the $\mathrm{sc}^{0}$-map $T^{k-1} f: T^{k-1} U \rightarrow T^{k-1} F$ is of class $\operatorname{sc}^{1}$. Its tangent map $T\left(T^{k-1} f\right)$ is then denoted by $T^{k} f$. It is an $\mathrm{sc}^{0}-$ map $T^{k} U \rightarrow T^{k} F$. A map which is of class $\mathrm{sc}^{k}$ for every $k$ is called sc-smooth or of class sc ${ }^{\infty}$.

Here are two useful observations which are proved in [6].

Proposition 2.14. If $f: U \subset E \rightarrow F$ is of class $s c^{k}$, then

$$
f: U_{m+k} \rightarrow F_{m}
$$

is of class $C^{k}$ for every $m \geq 0$.

If we denote the usual derivative of a map $f$ by $d f$ then for $x, h \in U_{m+1}$ we have the equality $d f(x)(h)=D f(x) h$. In fact, $D f(x)$ can be viewed as the (unique) continuous extension of $d f(x): E_{m+1} \rightarrow F_{m}$ to an operator $E \rightarrow F$ which satisfies $D f(x)\left(E_{m}\right) \subset$ $F_{m}$ for every $m \geq 0$ and induces continuous operators on these levels. It exists for every $x \in U_{1}$ due to the definition of the class $\mathrm{sc}^{1}$.

The second result is the following.

Proposition 2.15. Let $E$ and $F$ be sc-Banach spaces and let $U \subset E$ be open. Assume that the map $f: U \rightarrow F$ is $s c^{0}$ and that the induced map $f: U_{m+k} \rightarrow F_{m}$ is $C^{k+1}$ for every $m, k \geq 0$. Then $f: U \rightarrow E$ is sc-smooth. 
Reflecting on the notion of class $\mathrm{sc}^{1}$ one could expect for the composition $g \circ f$ of two such maps, that the target level would have to drop by 2 in order to obtain a $C^{1}$-map. In view of Proposition 2.14 one might think that therefore the composition needs not to be of class $\mathrm{sc}^{1}$. However, this is not the case, as the next result, the important chain rule shows.

Theorem 2.16 (Chain rule). Assume that $E, F$ and $G$ are sc-smooth Banach spaces and $U \subset E$ and $V \subset F$ are open sets. Assume that $f: U \rightarrow F, g: V \rightarrow G$ are of class $s c^{1}$ and $f(U) \subset V$. Then the composition $g \circ f: U \rightarrow G$ is of class $s^{1}$ and the tangent maps satisfy

$$
T(g \circ f)=T g \circ T f .
$$

Proof. We shall verify the properties (1) and (2) in Definition 2.13 for $g \circ f$. The functions $g: V_{1} \rightarrow G$ and $f: U_{1} \rightarrow F$ are of class $C^{1}$. Moreover, $D g(f(x)) \circ D f(x) \in \mathcal{L}(E, G)$ if $x \in U_{1}$. Fix $x \in U_{1}$ and choose $h \in E_{1}$ sufficiently small so that $f(x+h) \in V_{1}$. Then, using the postulated properties of $f$ and $g$, we have

$$
\begin{aligned}
g(f(x+h))-g(f(x))-D g(f(x)) \circ D f(x) h \\
=\quad \int_{0}^{1} D g(t f(x+h)+(1-t) f(x))[f(x+h)-f(x)-D f(x) h] d t \\
\quad+\int_{0}^{1}([D g(t f(x+h)+(1-t) f(x))-D g(f(x))] \circ D f(x) h) d t .
\end{aligned}
$$

Divide the first integral by the norm $\|h\|_{1}$; then

$$
\begin{aligned}
& \frac{1}{\|h\|_{1}} \int_{0}^{1} D g(t f(x+h)+(1-t) f(x))[f(x+h)-f(x)-D f(x) h] d t \\
& \quad=\int_{0}^{1} D g(t f(x+h)+(1-t) f(x)) \cdot \frac{1}{\|h\|_{1}}[f(x+h)-f(x)-D f(x) h] d t .
\end{aligned}
$$

If $h \in E_{1}$, the maps [0,1] $\rightarrow F_{1}$ defined by $t \mapsto t f(x+h)+(1-t) f(x)$ are continuous and converge in $C^{0}\left([0,1], F_{1}\right)$ to the constant map $t \mapsto f(x)$ as $\|h\|_{1} \rightarrow 0$. Moreover, since $f$ is of class $\mathrm{sc}^{1}$,

$$
a(h):=\frac{1}{\|h\|_{1}}[f(x+h)-f(x)-D f(x) h]
$$

converges to 0 in $F_{0}$ as $\|h\|_{1} \rightarrow 0$. Therefore, by the continuity assumption (2) in Definition 2.13, the map

$$
(t, h) \mapsto D g(t f(x+h)+(1-t) f(x))[a(h)]
$$

from $[0,1] \times E_{1}$ into $G_{0}$ converges to 0 as $h \rightarrow 0$, uniformly in $t$. Therefore, the expression in (3) converges to 0 in $G_{0}$ as $h \rightarrow 0$ in $E_{1}$. Next consider the integral

$$
\int_{0}^{1}[D g(t f(x+h)+(1-t) f(x))-D g(f(x))] \circ D f(x) \frac{h}{\|h\|_{1}} d t .
$$


In view of Definition 2.1 the set of all $h /\|h\|_{1} \in E_{1}$ has a compact closure in $E_{0}$. Therefore, since $D f(x) \in \mathcal{L}\left(E_{0}, F_{0}\right)$ is a continuous map by Definition 2.13 the closure of the set of all

$$
D f(x) \frac{h}{\|h\|_{1}}
$$

is compact in $F_{0}$. Consequently, again by Definition 2.1 every sequence $h_{n}$ converging to 0 in $E_{1}$ has a subsequence such that the integrand of the integral in (4) converges to 0 in $G_{0}$ uniformly in $t$. Hence the integral (4) also converges to 0 in $G_{0}$ as $h \rightarrow 0$ in $E_{1}$. We have proved that

$$
\frac{1}{\|h\|_{1}}\|g(f(x+h))-g(f(x))-D g(f(x)) \circ D f(x) h\|_{0} \rightarrow 0
$$

as $h \rightarrow 0$ in $E_{1}$. Consequently, condition (1) of Definition 2.13 is satisfied for the composition $g \circ f$ with the linear operator

$$
D(g \circ f)(x)=D g(f(x)) \circ D f(x) \in \mathcal{L}\left(E_{0}, G_{0}\right),
$$

where $x \in U_{1}$. We conclude that the tangent map $T(g \circ f): T U \rightarrow T G$,

$$
(x, h) \mapsto(g \circ f(x), D(g \circ f)(x) h),
$$

is sc-continuous and, moreover, $T(g \circ f)=T g \circ T f$. The proof of Theorem 2.16 is complete.

The reader should realize that in the above proof all conditions on $\mathrm{sc}^{1}$ maps have been used, i.e. it just works. From Theorem 2.16 one concludes by induction that the composition of two $\mathrm{sc}^{\infty}$-maps is also of class $\mathrm{sc}^{\infty}$ and, for every $k \geq 1$,

$$
T^{k}(g \circ f)=T^{k} g \circ T^{k} f .
$$

An sc-diffeomorphism $f: U \rightarrow V$, between open subsets $U$ and $V$ of sc-spaces $E$ and $F$ equipped with the induced sc-structure, is by definition a homeomorphism $U \rightarrow V$ so that $f$ and $f^{-1}$ are sc-smooth.

The following remark is a continuation of Remark 2.2

Remark 2.17. There are other possibilities for defining new concepts of smoothness. For example, we can drop the requirement of compactness of the embedding operator $E_{n} \rightarrow E_{m}$ for $n>m$. Then it is necessary to change the definition of smoothness in order to get the chain rule. One needs to replace the second condition in the definition of being $\mathrm{sc}^{1}$ by the requirement that $D f(x)$ induces a continuous linear operator $D f(x)$ : $E_{m-1} \rightarrow F_{m-1}$ for $x \in U_{m}$ and that the map $D f: U_{m} \rightarrow \mathcal{L}\left(E_{m-1}, F_{m-1}\right)$ for $m \geq 1$ is continuous. For this theory the sc-smooth structure on $E$ given by $E_{m}=E$ recovers the usual $C^{k}$-theory. However, this modified theory is not applicable to Gromov-Witten theory, Floer theory, and SFT. 


\subsection{Sc-manifolds}

Using the results so far, we can define sc-manifolds. This concept will not yet be sufficient to describe the spaces arising in SFT.

Definition 2.18. Let $X$ be a second countable Hausdorff space. An sc-chart of $X$ consists of a triple $(U, \varphi, E)$, where $U$ is an open subset of $X, E$ a Banach space with an sc-smooth structure and $\varphi: U \rightarrow E$ is a homeomorphism onto an open subset $V$ of E. Two such charts are sc-smoothly compatible provided the transition maps are scsmooth. An sc-smooth atlas consists of a family of charts whose domains cover $X$ so that any two charts are sc-smoothly compatible. A maximal sc-smooth atlas is called an scsmooth structure on $X$. The space $X$ equipped with a maximal sc-smooth atlas is called an sc-manifold.

Let us observe that a second countable Hausdorff space which admits an sc-smooth atlas is metrizable and paracompact since it is locally homeomorphic to open subsets of Banach spaces.

Assume that the space $X$ has an sc-smooth structure. Then it possesses the filtration $X_{m}$ for all $m \geq 0$ which is induced from the filtration of the charts. Moreover, every $X_{m}$ inherits the sc-smooth structure $\left(X_{m}\right)_{k}=X_{m+k}$ for all $k \geq 0$, denoted by $X^{m}$.

Next we shall define the tangent bundle $p: T X \rightarrow X^{1}$ in a natural way so that the tangent projection $p$ is sc-smooth. In order to do so, we use a modification of the definition found, for example, in Lang's book [14]. Namely, consider multiplets $(U, \varphi, E, x, h)$ where $(U, \varphi, E)$ is an sc-smooth chart, $x \in U_{1}$ and $h \in E$. Call two such tuples equivalent if $x=x^{\prime}$ and $D\left(\varphi^{\prime} \circ \varphi^{-1}\right)(\varphi(x)) h=h^{\prime}$. An equivalence class $[U, \varphi, E, x, h]$ is called a tangent vector at the point $x \in X_{1}$. The collection of all tangent vectors of $X$ is denoted by $T X$. The canonical projection is denoted by $p: T X \rightarrow X^{1}$. If $U \subset X$ is open we introduce the subset $T U \subset T X$ by $T U=p^{-1}\left(U \cap X^{1}\right)$. For a chart $(U, \varphi, E)$ we introduce the map

$$
T \varphi: T U \rightarrow E^{1} \oplus E
$$

defined by

$$
T \varphi([U, \varphi, E, x, h])=(x, h) .
$$

One easily checks that the collection of all triples $\left(T U, T \varphi, E^{1} \oplus E\right)$ defines an sc-smooth atlas for $T X$ for which the projection $p: T X \rightarrow X^{1}$ is an sc-smooth map. The tangent space $T_{X} X$ at $x \in X_{1}$ is the set of equivalence classes

$$
T_{x} X=\{[U, \varphi, E, x, h] \mid h \in E\}
$$

which inherits from $E$ the structure of a Banach space. If $x \in X_{m+1}$, then $T_{x} X$ has the partial filtration inherited from $E_{k}, 0 \leq k \leq m$. In particular, if $x \in X_{\infty}$, then $T_{x} X$ possesses an sc-smooth structure.

There is another class of bundles which can be defined in the present context. These are the so-called strong (vector) bundles. They may be viewed as a special case of strong M-polyfold bundles which will be introduced in Section 4 . For this reason we shall not introduce them separately and refer the reader to Remark 4.3 


\section{Splicing-based differential geometry}

In this section we introduce a 'splicing-based differential geometry'. The fundamental concepts are splicings and splicing cores. The splicing cores have locally varying dimensions but admit at the same time tangent spaces. Open subsets of the splicing cores will serve as the local models of the new global spaces called M-polyfolds. The letter M should remind us of a manifold type space obtained by gluing together the local models in an sc-smooth way. The M-polyfolds are equipped with substitutes for tangent bundles, so that one is able to linearize sc-smooth maps between M-polyfolds. In one of the follow-up papers we go further and introduce the notion of a polyfold which is a generalization of an orbifold and which is on the level of generalization needed for our applications.

\subsection{Quadrants and splicings}

Let us call a subset $C$ of an sc-Banach space $W$ a partial quadrant if there is an sc-Banach space $Q$ and a linear sc-isomorphism $T: W \rightarrow \mathbb{R}^{n} \oplus Q$ mapping $C$ onto $[0, \infty)^{n} \oplus Q$. If $Q=\{0\}$, then $C$ is called a quadrant. Observe that if $C$ and $C^{\prime}$ are partial quadrants, so is $C \oplus C^{\prime}$.

Definition 3.1. Assume $V$ is an open subset of a partial quadrant $C \subset W$. Let $E$ be an scBanach space and let $\pi_{v}: E \rightarrow E$, with $v \in V$, be a family of projections (i.e. $\pi_{v} \in \mathcal{L}(E)$ and $\pi_{v} \circ \pi_{v}=\pi_{v}$ ) so that the induced map

$$
\Phi: V \oplus E \rightarrow E, \quad \Phi(v, e)=\pi_{v}(e),
$$

is sc-smooth. Then the triple $\mathcal{S}=(\pi, E, V)$ is called an sc-smooth splicing.

The extension of the sc-smoothness definition of a map $f: V \rightarrow F$ from an open subset of an sc-Banach space to relatively open subsets $V \subset C \subset W$ of a partial quadrant $C$ in an sc-Banach space, which is used in Definition 3.1, is straightforward. One first observes that the sc-structure of $W$ induces a filtration on $V$. Now, the notion of the map $f$ to be an $\mathrm{sc}^{0}$-map is well defined. Then one defines an $\mathrm{sc}^{0}$-map $f: V \rightarrow F$ to be of class $\mathrm{sc}^{1}$ as in Definition 2.13 by replacing $U_{1}$ by $V_{1}$ there and requiring the existence of the limit for $x \in V_{1}$ and all $h \in W_{1}$ satisfying $x+h \in V_{1}$, with a linear map $D f(x) \in \mathcal{L}\left(W_{0}, F_{0}\right)$. Moreover, the tangent bundle $T V$ of the set $V$ is defined as usual by $T V=V^{1} \oplus W$ together with the $\mathrm{sc}^{0}$-projection map $T V \rightarrow V^{1}$.

We should point out that the sc-smoothness of the mapping $(v, e) \mapsto \pi_{v}(e)$ is a rather weak requirement allowing the dimension of the images of the projections $\pi_{v}$ to vary locally in the parameter $v \in V$. The reader can find illustrations and examples in [6].

Since $\pi_{v}$ is a projection,

$$
\Phi(v, \Phi(v, e))=\Phi(v, e) .
$$

The left hand side is the composition of $\Phi$ with the sc-smooth map $(v, e) \mapsto(v, \Phi(v, e))$. For fixed $(v, \delta v) \in T V$ we introduce the map

$$
P_{(v, \delta v)}: T E \rightarrow T E:(e, \delta e) \mapsto(\Phi(v, e), D \Phi(v, e)(\delta v, \delta e)) .
$$


It has the property that the induced map

$$
T V \oplus T E \rightarrow T E:(a, b) \mapsto P_{a}(b)
$$

is sc-smooth because, modulo the identification $T V \oplus T E=T(V \oplus E)$, it is equal to the tangent map of $\Phi$. From (5) one obtains by means of the chain rule (Theorem 2.16), at the points $(v, e) \in(V \oplus E)_{1}$, the formula

$$
D \Phi(v, \Phi(v, e))(\delta v, D \Phi(v, e)(\delta v, \delta e))=D \Phi(v, e)(\delta v, \delta e)
$$

and, together with the definition of $P$, one computes

$$
\begin{aligned}
P_{(v, \delta v)} \circ P_{(v, \delta v)}(e, \delta e) & =P_{(v, \delta v)}\left(\pi_{v}(e), D \Phi(v, e)(\delta v, \delta e)\right) \\
& =\left(\pi_{v}^{2}(e), D \Phi\left(v, \pi_{v}(e)\right)(\delta v, D \Phi(v, e)(\delta v, \delta e))\right) \\
& =\left(\pi_{v}(e), D \Phi(v, e)(\delta v, \delta e)\right)=P_{(v, \delta v)}(e, \delta e) .
\end{aligned}
$$

Consequently, $P_{(v, \delta v)}$ is a projection, which of course can be identified with the tangent $T \pi$ of the map $\pi: V \oplus E \rightarrow E$, defined by $\pi(v, e)=\pi_{v}(e)$, via

$$
P_{(v, \delta v)}(e, \delta e)=T \pi((v, e),(\delta v, \delta e)) .
$$

In the following we shall write $(T \pi)_{(v, \delta v)}$ instead of $P_{(v, \delta v)}$. Hence the triple

$$
T \mathcal{S}=(T \pi, T E, T V)
$$

is an sc-smooth splicing, called the tangent splicing of $\mathcal{S}$.

Definition 3.2. If $\mathcal{S}=(\pi, E, V)$ is an sc-smooth splicing, then the associated splicing core is the image bundle of the projection $\pi$ over $V$, i.e., it is the subset $K^{\mathcal{S}} \subset V \oplus E$ defined by

$$
K^{\mathcal{S}}:=\left\{(v, e) \in V \oplus E \mid \pi_{v}(e)=e\right\} .
$$

If the dimension of $E$ is finite, the images of the projections $\pi_{v}$ all have the same rank so that the splicing core is a smooth vector bundle over $V$. If, however, the dimension of $E$ is infinite, then the ranks of the fibers can change with the parameter $v$ thanks to the definition of sc-smoothness. This truly infinite-dimensional phenomenon is crucial for our purposes.

Every splicing $\mathcal{S}=(\pi, E, V)$ is accompanied by the complementary splicing $\mathcal{S}^{c}=$ $(1-\pi, E, V)$ where $1-\pi$ stands for the family of projections $\left(1-\pi_{v}\right)(e)=e-\pi_{v}(e)$ for $(v, e) \in V \oplus E$. This way the splicing decomposes the set $V \oplus E$ naturally into a fibered sum over the parameter set $V$. Indeed, $(v, e) \in V \oplus E$ can be decomposed as

$$
(v, e)=\left(v, e_{v}+e_{v}^{c}\right)
$$

where $\pi_{v}(e)=e_{v}$ and $\left(1-\pi_{v}\right)(e)=e_{v}^{c}$. The splicing cores $K^{\mathcal{S}}$ and $K^{\mathcal{S}^{c}}$ can be viewed as bundles over $V$ (with linear Banach space fibers, which however change dimensions). Their Whitney sum over $V$,

$$
K^{\mathcal{S}} \oplus_{V} K^{\mathcal{S}^{c}}=\left\{(v, a, b) \in V \oplus E \oplus E \mid \pi_{v}(a)=a, \pi_{v}(b)=0\right\},
$$


is naturally diffeomorphic to $V \oplus E$. The name splicing comes from the fact that it defines a decomposition of $V \oplus E \rightarrow V$, by 'splicing' it along $V$.

The splicing core of the tangent splicing $T \mathcal{S}$ is the set

$$
K^{T \mathcal{S}}=\left\{(v, \delta v, e, \delta e) \in T V \oplus T E \mid(T \pi)_{(v, \delta v)}(e, \delta e)=(e, \delta e)\right\} .
$$

The mapping

$$
K^{T \mathcal{S}} \rightarrow\left(K^{\mathcal{S}}\right)^{1}:(v, \delta v, e, \delta e) \mapsto(v, e) \in V_{1} \oplus E_{1}
$$

is the canonical projection. The fiber over every point $(v, e) \in\left(K^{\mathcal{S}}\right)^{1}$ is a subspace $K_{(v, e)}^{T \mathcal{S}}$ of the Banach space $W \oplus E$. If $(v, e)$ is on level $m+1$, then $K_{(v, e)}^{T \mathcal{S}}$ has well defined levels $k \leq m$. The tangent splicing $K^{T \mathcal{S}}$ has well defined bi-levels $(m, k)$ with $k \leq m$. Indeed, assume for simplicity that $W=\mathbb{R}^{n} \oplus Q$; then $V \subset C \subset W$ and $T V=V^{1} \oplus W$ and we can define for $0 \leq k \leq m$,

$$
\left(K^{T \mathcal{S}}\right)_{m, k}=\left\{(v, \delta v, e, \delta e) \in V_{m+1} \oplus W_{k} \oplus E_{m+1} \oplus E_{k} \mid(T \pi)_{(v, \delta v)}(e, \delta e)=(e, \delta e)\right\} .
$$

The projection $K^{T \mathcal{S}} \rightarrow\left(K^{\mathcal{S}}\right)^{1}:(v, \delta v, e, \delta e) \mapsto(v, e)$ maps level $(m, k)$ points to level $m$ points. We may view $k$ as the fiber regularity and $m$ as the base regularity. Note that a point $e$ of $E^{j}$ of regularity $m$ has regularity $m+j$ as a point in $E$. The following is one of our main definitions.

Definition 3.3. A local M-polyfold model consists of a pair $(O, \mathcal{S})$ where $O$ is an open subset of the splicing core $K^{\mathcal{S}} \subset V \oplus E$ associated with the sc-smooth splicing $\mathcal{S}=$ $(\pi, E, V)$. The tangent of the local M-polyfold model $(O, \mathcal{S})$ is the object defined by

$$
T(O, \mathcal{S})=\left(K^{T \mathcal{S}} \mid O^{1}, T \mathcal{S}\right)
$$

where $K^{T \mathcal{S}} \mid O^{1}$ denotes the collection of all points in $K^{T \mathcal{S}}$ which project under the canonical projection $K^{T \mathcal{S}} \rightarrow\left(K^{\mathcal{S}}\right)^{1}$ onto the points in $O^{1}$.

There is the natural projection

$$
K^{T \mathcal{S}_{\mid} O^{1} \rightarrow O^{1}:(v, \delta v, e, \delta e) \mapsto(v, e) .}
$$

In the following we shall simply write $O$ instead of $(O, \mathcal{S})$, but keep in mind that $\mathcal{S}$ is part of the structure. With this notation the tangent $T O=T(O, \mathcal{S})$ of the open subset $O$ of the splicing core $K^{\mathcal{S}}$ is the set

$$
T O=K^{T \mathcal{S}} \mid O^{1} .
$$

Note that on an open subset $O$ of a splicing core there is an induced filtration. Hence we may talk about sc ${ }^{0}$-maps. We will see in the next section that there is also a well defined notion of an $\mathrm{sc}^{1}$-map in this setting. We shall see in the applications presented in [6, 7, 11, [12] that analytical limiting phenomena, like bubbling-off, occurring in symplectic field theory, Gromov-Witten theory and Floer theory are smooth within the splicing world. 


\subsection{Smooth maps between splicing cores}

The aim of this section is to introduce the concept of an $\mathrm{sc}^{1}$-map between local Mpolyfold models. We will construct the tangent functor and show the validity of the chain rule. At that point we will have established all the ingredients for building the 'splicingdifferential geometry' mentioned in the introduction.

Consider two open subsets $O \subset K^{\mathcal{S}} \subset V \oplus E$ and $O^{\prime} \subset K^{\mathcal{S}^{\prime}} \subset V^{\prime} \oplus E^{\prime}$ of splicing cores belonging to the splicings $\mathcal{S}=(\pi, E, V)$ and $\mathcal{S}^{\prime}=\left(\pi^{\prime}, E^{\prime}, V^{\prime}\right)$. The open subsets $V$ and $V^{\prime}$ of partial quadrants are contained in the sc-Banach spaces $W$ resp. $W^{\prime}$. Consider an $\mathrm{sc}^{0}$-map $f: O \rightarrow O^{\prime}$.

If $O$ is an open subset of the splicing core $K^{\mathcal{S}} \subset V \oplus E$ we define the subset $\widehat{O}$ of $V \oplus E$ by

$$
\widehat{O}=\left\{(v, e) \in V \oplus E \mid\left(v, \pi_{v}(e)\right) \in O\right\} .
$$

Clearly, $\widehat{O}$ is open in $V \oplus E$ and can be viewed as a bundle $\widehat{O} \rightarrow O$ over $O$. This bundle will be important in Subsection 4.2 , where the crucial notion of a filler is introduced.

Definition 3.4. The $s c^{0}$-continuous map $f: O \rightarrow O^{\prime}$ between open subsets of splicing cores is called of class $\mathrm{sc}^{1}$ if the map

$$
\widehat{f}: \widehat{O} \subset V \oplus E \rightarrow W^{\prime} \oplus E^{\prime}, \quad \widehat{f}(v, e)=f\left(v, \pi_{v}(e)\right),
$$

is of class $\mathrm{sc}^{1}$.

According to the splitting of the image space we set

$$
\widehat{f}(v, e)=\left(\widehat{f_{1}}(v, e), \widehat{f_{2}}(v, e)\right) \in K^{\mathcal{S}^{\prime}} \subset W^{\prime} \oplus E^{\prime} .
$$

The tangent map $T \widehat{f}$ associated with the $\mathrm{sc}^{1}$-map $\widehat{f}$ is defined as

$$
T \widehat{f}(v, \delta v, e, \delta e):=\left(T \widehat{f_{1}}(v, \delta v, e, \delta e), T \widehat{f_{2}}(v, \delta v, e, \delta e)\right) .
$$

The map $T \widehat{f}$ is of class $\mathrm{sc}^{0}$.

Lemma 3.5. The tangent map $T \widehat{f}$ satisfies $T \widehat{f}\left(K^{T \mathcal{S}} \mid O^{1}\right) \subset K^{T \mathcal{S}^{\prime}} \mid O^{\prime 1}$ and hence induces a map

$$
K^{T \mathcal{S}}\left|O^{1} \rightarrow K^{T \mathcal{S}^{\prime}}\right| O^{\prime 1}
$$

which we denote by $T f$. In the simplified notation of (9), we have

$$
T f: T O \rightarrow T O^{\prime}
$$

Proof. Denote by $\pi_{v^{\prime}}^{\prime}$ the family of projections associated with the splicing $\mathcal{S}^{\prime}$. Since $f: O \rightarrow O^{\prime}$, by definition of the splicing core $K^{\mathcal{S}^{\prime}}$ we have the formula

$$
\pi^{\prime} \widehat{f}_{1}(v, e)\left(\widehat{f_{2}}(v, e)\right)=\widehat{f_{2}}(v, e)
$$


Differentiating this identity in the variable $(v, e)$ we obtain

$$
\begin{aligned}
D \widehat{f_{2}}(v, e)(\delta v, \delta e)= & D_{v^{\prime}} \pi_{\widehat{f}_{1}(v, e)}^{\prime}\left(\widehat{f_{2}}(v, e)\right) \circ D \widehat{f_{1}}(v, e)(\delta v, \delta e) \\
& +\pi_{\widehat{f}_{1}(v, e)}^{\prime} \circ D \widehat{f_{2}}(v, e)(\delta v, \delta e) .
\end{aligned}
$$

Set

$$
v^{\prime}=\widehat{f_{1}}(v, e), e^{\prime}=\widehat{f_{2}}(v, e), \delta v^{\prime}=D \widehat{f_{1}}(v, e)(\delta v, \delta e), \delta e^{\prime}=D \widehat{f_{2}}(v, e)(\delta v, \delta e) .
$$

Then (11) implies using the definition (6) of the projection $\left(T \pi^{\prime}\right)_{\left(v^{\prime}, \delta v^{\prime}\right)}$ associated with the splicing $T \mathcal{S}^{\prime}$ that

$$
\left(T \pi^{\prime}\right)_{\left(v^{\prime}, \delta v^{\prime}\right)}\left(e^{\prime}, \delta e^{\prime}\right)=\left(e^{\prime}, \delta e^{\prime}\right) .
$$

So indeed $T f(v, \delta v, e, \delta e)=\left(v^{\prime}, \delta v^{\prime}, e^{\prime}, \delta e^{\prime}\right) \in K^{T \mathcal{S}^{\prime}}$, as claimed.

Note that the order of the terms in the tangent map $T f$ resp. $T \widehat{f}$ of an $\operatorname{sc}^{1}$-map $f$ : $O \rightarrow O^{\prime}$ is different from the order of terms in the classical notation. Writing $f=$ $\left(f_{1}, f_{2}\right)$ according to the splitting of the image space into the distinguished splicing parameter part and the standard part, the classical notation for the tangent map would be $T f=\left(\left(f_{1}, f_{2}\right),\left(D f_{1}, D f_{2}\right)\right)$ whereas our convention is $T f=\left(\left(f_{1}, D f_{1}\right),\left(f_{2}, D f_{2}\right)\right)$. This rather unorthodox ordering of the data has been chosen so that the tangent of a splicing is again a splicing.

The reader could work out as an example the situation where the splicings have the constant projection Id.

Theorem 3.6 (Chain rule). Let $O, O^{\prime}, O^{\prime \prime}$ be open subsets of splicing cores and let the maps $f: O \rightarrow O^{\prime}$ and $g: O^{\prime} \rightarrow O^{\prime \prime}$ be of class $s c^{1}$. Then the composition $g \circ f$ is also of class $\mathrm{sc}^{1}$ and the tangent map satisfies

$$
T(g \circ f)=T g \circ T f .
$$

Proof. This is a consequence of the sc-chain rule (Theorem 2.16), the definition of the tangent map and the fact that our reordering of the terms in our definition 10 of the tangent map is consistent. Indeed, from definition (10) we deduce

$$
\begin{aligned}
T(g \circ f)(v, \delta v, e, \delta e) & =\left(T\left(\widehat{g}_{1} \circ \widehat{f}\right)(v, e, \delta v, \delta e), T\left(\widehat{g}_{2} \circ \widehat{f}\right)(v, e, \delta v, \delta e)\right) \\
& =\left(\left(T \hat{g}_{1}\right) \circ(T \hat{f})(v, e, \delta v, \delta e),\left(T \widehat{g}_{2}\right) \circ(T \widehat{f})(v, e, \delta v, \delta e)\right) \\
& =(T g)\left(T \widehat{f}_{1}(v, e, \delta v, \delta e), T \widehat{f}_{2}(v, e, \delta v, \delta e)\right) \\
& =(T g) \circ(T f)(v, \delta v, e, \delta e)
\end{aligned}
$$

and the proof is complete.

Given an $\operatorname{sc}^{1}$-map $f: O \rightarrow O^{\prime}$ between open sets of splicing cores we obtain, in view of Lemma 3.5. an induced tangent map $T f: T O \rightarrow T O^{\prime}$. Since $T O$ and $T O^{\prime}$ are again open sets in the splicing cores $K^{T \mathcal{S}}$ and $K^{T \mathcal{S}^{\prime}}$ we can iteratively define the notion of $f$ to be of class $s c^{k}$ and of $f$ to be sc-smooth. 
Definition 3.7. Let $O$ be an open subset of a splicing core $K^{\mathcal{S}}$ and $(v, e) \in O_{1}$. The tangent space to $O$ at the point $(v, e)$ is the Banach space

$$
T_{(v, e)} O=\{(\delta v, \delta e) \in W \oplus E \mid(v, \delta v, e, \delta e) \in T O\} .
$$

We then have

$$
T O=\bigcup_{(v, e) \in O_{1}} T_{(v, e)} O
$$

If $f: O \rightarrow O^{\prime}$ is a homeomorphism so that $f$ and $f^{-1}$ are sc-smooth, our tangent map $T f$ defined in 10 induces the linear isomorphism

$$
T f(v, e): T_{(v, e)} O \rightarrow T_{f(v, e)} O^{\prime} .
$$

We recall from Section 3.1 that the space $T O$ has a bi-filtration $(T O)_{(m, k)}$ for $0 \leq k \leq m$, so that the natural projection

$$
T O \rightarrow O^{1}
$$

maps level $(m, k)$ points to level $m$ points and is sc-smooth. The projection map $T O \rightarrow$ $O^{1}$ is sc-smooth.

\subsection{M-polyfolds}

Now we are able to introduce the notion of an M-polyfold. The "M" indicates the "manifold flavor' of the polyfold. A general polyfold will be a generalization of an orbifold.

Definition 3.8. Let $X$ be a second countable Hausdorff space. An M-polyfold chart for $X$ is a triple $(U, \varphi, \mathcal{S})$, in which $U$ is an open subset of $X, \mathcal{S}=(\pi, E, V)$ an sc-smooth splicing and $\varphi: U \rightarrow K^{\mathcal{S}}$ a homeomorphism onto an open subset $O$ of the splicing core $K^{\mathcal{S}}$ of $\mathcal{S}$. Two charts are called compatible if the transition maps between open subsets of splicing cores are sc-smooth in the sense of Definition 3.4 A maximal atlas of sc-smoothly compatible M-polyfold charts is called an M-polyfold structure on X.

An M-polyfold is necessarily metrizable by an argument similar to the one used already for sc-manifolds. Each splicing core $K^{\mathcal{S}}$ carries the structure of an M-polyfold with the global chart being the identity.

The concept of a map $f: X \rightarrow X^{\prime}$ between M-polyfolds being of class $\mathrm{sc}^{0} \mathrm{or} \mathrm{sc}^{k}$ or being sc-smooth is, as usual, defined by means of local charts.

Definition 3.9. The mapping $f: X \rightarrow X^{\prime}$ between two M-polyfolds is called of class $\mathrm{sc}^{0}$ resp. $\mathrm{sc}^{\mathrm{k}}$ or called $\mathrm{sc}-\mathrm{smooth}$ if for every point $x \in X$ there exists a chart $(U, \varphi, \mathcal{S})$ around $x$ and a chart $\left(U^{\prime}, \varphi^{\prime}, \mathcal{S}^{\prime}\right)$ around $f(x)$ so that $f(U) \subset U^{\prime}$ and

$$
\varphi^{\prime} \circ f \circ \varphi^{-1}: \varphi(U) \rightarrow \varphi^{\prime}\left(U^{\prime}\right)
$$

is of class $s c^{0}$ resp. $s c^{k}$ or sc-smooth. 
In order to define the tangent space $T_{x} X$ of the M-polyfold $X$ at the point $x \in X_{1}$, we proceed as in the case of sc-manifolds in Section 3.3. This time we consider equivalence classes of multiplets $(U, \varphi, S, x, h)$ in which $(U, \varphi, S)$ is an M-polyfold chart, $x$ is a point in $U_{1}$ and $h \in T_{\varphi(x)} O$, where $O=\varphi(U) \subset K^{\mathcal{S}}$ is the open set of the splicing core. The above multiplet is equivalent to $\left(U^{\prime}, \varphi^{\prime}, S^{\prime}, x^{\prime}, h^{\prime}\right)$ if $x=x^{\prime}$ and if $T\left(\varphi^{\prime} \circ \varphi^{-1}\right)(\varphi(x)) h$ $=h^{\prime}$, where the tangent map

$$
T\left(\varphi^{\prime} \circ \varphi^{-1}\right)(\varphi(x)): T_{\varphi(x)} O \rightarrow T_{\varphi^{\prime}(x)} O^{\prime}
$$

is defined in Section 3.2. The tangent space is now defined as the set of equivalence classes

$$
T_{x} X=\left\{[U, \varphi, S, x, h] \mid h \in T_{\varphi(x)} O\right\} .
$$

It inherits the structure of a Banach space from the tangent space $T_{\varphi(x)} O$. If $x \in X_{m+1}$, then $T_{X} X$ possesses a partial filtration for $0 \leq k \leq m$ induced from the partial filtration of $T_{\varphi(x)} O$. The tangent space at a smooth point $x \in X_{\infty}$ possesses an sc-smooth structure.

Let now $f: X \rightarrow X^{\prime}$ be a map between M-polyfolds of class $\operatorname{sc}^{k}$ for $k \geq 1$. In two M-polyfold charts $(U, \varphi, S)$ and $\left(U^{\prime}, \varphi^{\prime}, S^{\prime}\right)$ around the points $x \in U_{1}$ and $f(x) \in U_{1}^{\prime}$, the map $f$ is represented by the $\operatorname{sc}^{k}$-map $\psi: \varphi^{\prime} \circ f \circ \varphi^{-1}: O \rightarrow O^{\prime}$ between open sets of splicing cores. The tangent map $T \psi(x): T_{\varphi(x)} O \rightarrow T_{\psi(x)} O^{\prime}$ defines a unique continuous linear map

$$
T_{x} f: T_{x} X \rightarrow T_{f(x)} X^{\prime}
$$

between the tangent spaces, called the tangent map of $f$ at the point $x$, mapping the equivalence class $[U, \varphi, S, x, h]$ into the class $\left[U^{\prime}, \varphi^{\prime}, S^{\prime}, f(x), h^{\prime}\right]$ in which

$$
h^{\prime}=T\left(\varphi^{\prime} \circ f \circ \varphi^{-1}\right)(\varphi(x)) \cdot h .
$$

If $x$ is a smooth point of $X$ and if $f$ is an sc-smooth map, then the tangent map $T_{x} f$ is an sc-operator as defined in Section 2.2

Let us note the following useful result about sc-smooth partitions of unity.

Theorem 3.10. Let $X$ be an M-polyfold with local models being splicing cores build on separable sc-Hilbert spaces. (An sc-Hilbert space consists of a Hilbert space equipped with an sc-structure. It is not required that the Banach spaces $E_{m}$ for $m \geq 1$ are Hilbert spaces.) Assume that $\left(U_{\lambda}\right)_{\lambda \in \Lambda}$ is an open covering of $X$. Then there exists a subordinate sc-smooth partition of unity $\left(\beta_{\lambda}\right)_{\lambda \in \Lambda}$.

The statement follows along the lines of a proof for Hilbert manifolds in [14]. The product $X \times Y$ of two M-polyfolds is in a natural way an M-polyfold. Indeed, if $(U, \varphi, \mathcal{S})$ and $(W, \psi, \mathcal{T})$ are M-polyfold charts for $X$ and $Y$ respectively, one obtains the product chart $(U \times W, \varphi \times \psi, \mathcal{S} \times \mathcal{T})$ for $X \times Y$, with the product splicing

$$
\mathcal{S} \times \mathcal{T}=(\pi, E, V) \times\left(\rho, F, V^{\prime}\right)=\left(\sigma, E \oplus F, V \oplus V^{\prime}\right)
$$

where $\sigma_{\left(v, v^{\prime}\right)}=\pi_{v} \oplus \rho_{v^{\prime}}$ is the family of projections. There are several possible notions of sub-polyfolds (we suppress the $M$ in the notation). We shall describe one of them in Section 3.5 below and refer the reader to [6] for a comprehensive treatment. 


\subsection{Corners and boundary points}

In this section we will prove the extremely important fact that sc-smooth maps are able to recognize corners. This will be crucial for the SFT because most of its algebraic structure is a consequence of the corner structure.

Let $X$ be an M-polyfold. Around a point $x \in X$ we take an M-polyfold chart $\varphi$ : $U \rightarrow K^{\mathcal{S}}$ where $K^{\mathcal{S}}$ is the splicing core associated with the splicing $\mathcal{S}=(\pi, E, V)$. Here $V$ is an open subset of a partial quadrant $C$ contained in the sc-Banach space $W$. By definition there exists a linear isomorphism from $W$ to $\mathbb{R}^{n} \oplus Q$ mapping $C$ onto $[0, \infty)^{n} \oplus Q$. Identifying the partial quadrant $C$ with $[0, \infty)^{n} \oplus Q$ we shall use the notation $\varphi=\left(\varphi_{1}, \varphi_{2}\right) \in[0, \infty)^{n} \oplus(Q \oplus E)$ according to the splitting of the target space of $\varphi$. We associate with the point $x \in U$ the integer $d(x)$ defined by

$$
d(x)=\sharp\left\{\text { coordinates of } \varphi_{1}(x) \text { which are equal to } 0\right\} .
$$

Theorem 3.11. The map $d: X \rightarrow \mathbb{N}$ is well defined and does not depend on the choice of the M-polyfold chart $\varphi: U \rightarrow K^{\mathcal{S}}$. Moreover, every point $x \in X$ has an open neighborhood $U^{\prime}$ satisfying

$$
d(y) \leq d(x) \quad \text { for all } y \in U^{\prime} .
$$

Definition 3.12. The map $d: X \rightarrow \mathbb{N}$ is called the degeneracy index of $X$.

The map $d$ will play an important role in our Fredholm theory with operations presented in [10]. A point $x \in X$ satisfying $d(x)=0$ is called an interior point. A point satisfying $d(x)=1$ is called a good boundary point. A point with $d(x) \geq 2$ is called a corner. In general, the integer $d(x)$ is the order of the corner.

Proof of Theorem 3.11. Consider two M-polyfold charts $\varphi: \widehat{U} \subset X \rightarrow K^{S}$ and $\varphi^{\prime}$ : $\widehat{U}^{\prime} \subset X \rightarrow K^{\mathcal{S}^{\prime}}$ such that $x \in \widehat{U} \cap \widehat{U}^{\prime}$. Introducing the open subsets $U=\varphi\left(\widehat{U} \cap \widehat{U}^{\prime}\right)$ and $U^{\prime}=\varphi^{\prime}\left(\widehat{U} \cap \widehat{U}^{\prime}\right)$ of $K^{\mathcal{S}}$ and $K^{\mathcal{S}^{\prime}}$ resp., and setting $\varphi(x)=(r, a)$ and $\varphi^{\prime}(x)=\left(r^{\prime}, a^{\prime}\right)$ we define the sc-diffeomorphism $\Phi: U \rightarrow U^{\prime}$ by $\Phi=\varphi^{\prime} \circ \varphi^{-1}$. Obviously, $\Phi(r, a)=$ $\left(r^{\prime}, a^{\prime}\right)$. Now the proof of Theorem 3.11 reduces to the following proposition.

Proposition 3.13. Let $\mathcal{S}=(\pi, E, V)$ and $\mathcal{S}^{\prime}=\left(\pi^{\prime}, E^{\prime}, V^{\prime}\right)$ be two splicings having the parameter sets $V=[0, \infty)^{k} \oplus Q$ and $V^{\prime}=[0, \infty)^{k^{\prime}} \oplus Q^{\prime}$. Assume that $U$ and $U^{\prime}$ are open subsets of the splicing cores $K^{\mathcal{S}}$ and $K^{\mathcal{S}^{\prime}}$ containing the points $(r, a)$ and $\left(r^{\prime}, a^{\prime}\right)$ with $r \in[0, \infty)^{k}$ and $r^{\prime} \in[0, \infty)^{k^{\prime}}$ and assume that the map $\Phi: U \rightarrow U^{\prime}$ is an sc-diffeomorphism mapping $(r, a)$ to $\left(r^{\prime}, a^{\prime}\right)$. Then $r$ and $r^{\prime}$ have the same number of vanishing coordinates.

Proof. We first prove the assertion under the additional assumption that the point $p_{0}=$ $(r, a)$ belongs to $U_{\infty}$. Then the image point $q_{0}=\left(r^{\prime}, a^{\prime}\right)=\Phi\left(p_{0}\right)$ belongs to $U_{\infty}^{\prime}$. Denote by $J$ the subset of $\{1, \ldots, k\}$ consisting of those indices $j$ for which $r_{j}=0$. Similarly, $j^{\prime} \in J^{\prime} \subset\left\{1, \ldots, k^{\prime}\right\}$ if $r_{j}^{\prime}=0$. Denoting by $\sharp r$ and $\sharp r^{\prime}$ the cardinalities of $J$ and $J^{\prime}$ we claim that $\sharp r=\sharp r^{\prime}$. Since $\Phi$ is an sc-diffeomorphism it suffices to prove the inequality $\sharp r \geq \sharp r^{\prime}$ since this inequality also has to hold true for the sc-diffeomorphism $\Phi^{-1}$. Write 
$a=(q, e)$. If $\pi_{(r, q)}(e)=e$, then differentiating $\pi_{(r, q)} \circ \pi_{(r, q)}(e)=\pi_{(r, q)}(e)$ in $(r, q)$ one finds $\pi_{(r, q)} \circ D_{(r, q)}\left(\pi_{(r, q)}(e)\right)=0$ so that $D_{(r, q)}\left(\pi_{r}(e)\right)(\delta r, \delta q)$ is contained in the range of Id $-\pi_{(r, q)}$. Therefore, given $(r, a) \in U_{\infty}$ satisfying $\pi_{(r, q)}(e)=e$ and given $\delta r \in \mathbb{R}^{k}$ and $\delta q \in Q_{\infty}$, there exists $\delta e \in E_{\infty}$ solving

$$
\delta e=\pi_{(r, q)}(\delta e)+D_{(r, q)}\left(\pi_{(r, q)}(e)\right)[(\delta r, \delta q)] .
$$

In particular, taking $\delta r \in \mathbb{R}^{k}$ with $(\delta r)_{j}=0$ for $j \in J$, and a smooth $\delta q$, there exists $\delta e \in E_{\infty}$ solving the equation (14). This is equivalent to $((\delta r, \delta q), \delta e) \in\left(T_{(r, a)} U\right)_{\infty}$. Introduce the path

$$
\tau \mapsto p_{\tau}=\left(r+\tau \delta r, q+\tau \delta q, \pi_{(r+\tau \delta r, q+\tau \delta q)}(e+\tau \delta e)\right)
$$

for $|\tau|<\rho$ and $\rho$ small. From $(r, a) \in U_{\infty}$ and $\delta e \in E_{\infty}$ one concludes $p_{\tau} \in U_{\infty}$. Moreover, if we consider $\tau \mapsto p_{\tau}$ as a map into $U_{m}$ for $m \geq 0$, its derivative at $\tau=0$ is equal to $(\delta r, \delta q, \delta e)$. Fix a level $m \geq 1$ and consider for $\rho>0$ sufficiently small the map

$$
(-\rho, \rho) \rightarrow \mathbb{R}^{k^{\prime}} \oplus Q_{m}^{\prime} \oplus E_{m}^{\prime}: \tau \mapsto \Phi\left(p_{\tau}\right) .
$$

The map $\Phi: U \rightarrow U^{\prime}$ is $C^{1}$ as a map from $U_{m+1} \subset \mathbb{R}^{k} \oplus Q_{m+1} \oplus E_{m+1}$ into $\mathbb{R}^{k^{\prime}} \oplus Q_{m}^{\prime} \oplus$ $E_{m}^{\prime}$. Its derivative $d \Phi(r, q, e): \mathbb{R}^{k} \oplus Q_{m+1} \oplus E_{m+1} \rightarrow \mathbb{R}^{k^{\prime}} \oplus Q_{m}^{\prime} \oplus E_{m}^{\prime}$ has an extension to a continuous linear operator $D \Phi(r, q, e): \mathbb{R}^{k} \oplus Q_{m} \oplus E_{m} \rightarrow \mathbb{R}^{k^{\prime}} \oplus Q_{m}^{\prime} \oplus E_{m}^{\prime}$. Since $\Phi$ is an sc-diffeomorphism the extension $D \Phi(r, q, e): \mathbb{R}^{k} \oplus Q_{m} \oplus E_{m} \rightarrow \mathbb{R}^{k^{\prime}} \oplus Q_{m}^{\prime} \oplus E_{m}^{\prime}$ is a bijection. Thus, since $\delta q \in Q_{\infty}$ and $\delta e \in E_{\infty}$,

$$
\begin{aligned}
\Phi\left(p_{\tau}\right) & =\Phi\left(p_{0}\right)+\tau \cdot d \Phi\left(p_{0}\right)[\delta r, \delta q, \delta e]+o_{m}(\tau) \\
& =q_{0}+\tau \cdot D \Phi\left(p_{0}\right)[\delta r, \delta q, \delta e]+o_{m}(\tau)
\end{aligned}
$$

where $o_{m}(\tau)$ is a function taking values in $\mathbb{R}^{k^{\prime}} \oplus Q_{k}^{\prime} \oplus E_{m}^{\prime}$ and satisfying $\tau^{-1} o_{m}(\tau) \rightarrow 0$ as $\tau \rightarrow 0$. Introduce the sc-continuous linear functionals $\lambda_{j^{\prime}}: \mathbb{R}^{k^{\prime}} \oplus Q^{\prime} \oplus E^{\prime} \rightarrow \mathbb{R}$ by

$$
\lambda_{j^{\prime}}\left(s^{\prime}, q^{\prime}, h^{\prime}\right)=s_{j^{\prime}}^{\prime}
$$

where $j^{\prime} \in\left\{1, \ldots, k^{\prime}\right\}$. Then

$$
\lambda_{j^{\prime}} \circ \Phi\left(p_{\tau}\right) \geq 0
$$

for $|\tau|<\rho$ and $j^{\prime} \in\left\{1, \ldots, k^{\prime}\right\}$. Applying for $j^{\prime} \in J^{\prime}$ the functional $\lambda_{j^{\prime}}$ to both sides of (15) and using the fact that $\lambda_{j^{\prime}}\left(\Phi\left(p_{0}\right)\right)=\lambda_{j^{\prime}}\left(q_{0}\right)=0$ for $j^{\prime} \in J^{\prime}$ we conclude that for $\tau>0$,

$$
\begin{aligned}
0 \leq \frac{1}{\tau} \cdot \lambda_{j^{\prime}}\left[\Phi\left(p_{\tau}\right)\right] & =\frac{1}{\tau} \cdot \lambda_{j^{\prime}}\left[\Phi\left(p_{0}\right)+\tau \cdot D \Phi\left(p_{0}\right)[\delta r, \delta q, \delta e]+o_{m}(\tau)\right] \\
& =\lambda_{j^{\prime}}\left[D \Phi\left(p_{0}\right)[\delta r, \delta q, \delta e]\right]+\lambda_{j^{\prime}}\left(\frac{o_{m}(\tau)}{\tau}\right) .
\end{aligned}
$$

Passing to the limit $\tau \rightarrow 0^{+}$we find

$$
0 \leq \lambda_{j^{\prime}}\left(D \Phi\left(p_{0}\right)[\delta r, \delta q, \delta e]\right)
$$


and replacing $(\delta r, \delta q, \delta e)$ by $(-\delta r,-\delta a,-\delta e)$ we obtain the equality sign. Consequently,

$$
\lambda_{j^{\prime}}\left(D \Phi\left(p_{0}\right)[\delta r, \delta q, \delta e]\right)=0, \quad j^{\prime} \in J^{\prime},
$$

for all $[\delta r, \delta q, \delta e] \in \mathbb{R}^{k} \oplus Q_{\infty} \oplus E_{\infty}$ satisfying

$$
\pi_{(r, q)}(\delta e)+D_{(r, q)}\left(\pi_{(r, q)}(e)\right)[(\delta r, \delta q)]=\delta e
$$

and $(\delta r)_{j}=0$ for all $j \in J$. Introduce the codimension $\sharp r$ subspace $L$ of the tangent space $T_{(r, q, e)} U \subset K^{T \mathcal{S}}$ which we may view as a subset of $\mathbb{R}^{k} \oplus Q_{\infty} \oplus E_{\infty}$ by

$$
\begin{array}{r}
L=\left\{(\delta r, \delta q, \delta e) \in \mathbb{R}^{k} \oplus Q_{\infty} \oplus E_{\infty} \mid \pi_{(r, q)}(\delta e)+D_{(r, q)}\left(\pi_{(r, q)}(e)\right)(\delta r, \delta q)=\delta e\right. \\
\text { and } \left.(\delta r)_{j}=0 \text { for all } j \in J\right\} .
\end{array}
$$

Then, in view of $(16)$,

$$
\begin{array}{r}
D \Phi(r, q, e) L \subset\left\{\left(\delta r^{\prime}, \delta q^{\prime}, \delta e^{\prime}\right) \mid \pi_{\left(r^{\prime}, q^{\prime}\right)}^{\prime}\left(\delta e^{\prime}\right)+D_{\left(r^{\prime}, q^{\prime}\right)}\left(\pi_{\left(r^{\prime}, q^{\prime}\right)}^{\prime}\left(e^{\prime}\right)\right)\left(\delta r^{\prime}, \delta q^{\prime}\right)=\delta e^{\prime}\right. \\
\text { and } \left.\left(\delta r^{\prime}\right)_{j^{\prime}}=0 \text { for all } j^{\prime} \in J^{\prime}\right\} .
\end{array}
$$

Because the subspace on the right hand side has codimension $\sharp r^{\prime}$ in $T_{\left(r^{\prime}, q^{\prime}, e^{\prime}\right)} U^{\prime}$ and since $D \Phi(r, q, e)$, being a bijection, maps $L$ onto a codimension $\sharp r$ subspace of $T_{\left(r^{\prime}, q^{\prime}, e^{\prime}\right)} U^{\prime}$, it follows that $\sharp r^{\prime} \leq \sharp r$, as claimed.

Next we shall prove the general case. For this we take $p_{0}=(r, q, e)$ in $U_{0}$, so that the image point $\left(r^{\prime}, q^{\prime}, e^{\prime}\right)=\Phi(r, q, e)$ belongs to $U_{0}^{\prime}$. Arguing by contradiction we may assume that $\sharp r>\sharp r^{\prime}$, otherwise we replace $\Phi$ by $\Phi^{-1}$. Since $U_{\infty}$ is dense in $U_{0}$ we find a sequence $\left(r, q_{n}, e_{n}\right) \in U_{\infty}$ satisfying $\pi_{\left(r, q_{n}\right)}\left(e_{n}\right)=e_{n}$ and $\left(r, q_{n}, e_{n}\right) \rightarrow(r, q, e)$ in $U_{0}$. By the previous discussion $\sharp r=\sharp r_{n}^{\prime}$ where $\left(r_{n}^{\prime}, q_{n}^{\prime}, e_{n}^{\prime}\right)=\Phi\left(r, q_{n}, e_{n}\right)$. Since $\Phi$ is sc-smooth, we have $\left(r_{n}^{\prime}, q_{n}^{\prime}, e_{n}^{\prime}\right) \rightarrow\left(r^{\prime}, q^{\prime}, e^{\prime}\right)$ in $U_{0}^{\prime}$ and $\pi_{\left(r^{\prime}, q^{\prime}\right)}^{\prime}\left(e^{\prime}\right)=e^{\prime}$. From this convergence we deduce $\sharp r^{\prime} \geq \sharp r_{n}^{\prime}$ so that $\sharp r^{\prime} \geq \sharp r$, contradicting our assumption. The proof of Proposition 3.13 is complete.

To finish the proof of Theorem 3.11 it remains to show that the function $d$ is lower semicontinuous. Assume for the moment that there exists a sequence of points $x_{k}$ converging to $x$ so that $d\left(x_{k}\right)>d(x)$. Since $\varphi$ is continuous, we have the convergence $\varphi_{1}\left(x_{k}\right)=\left(r_{1}^{k}, \ldots, r_{n}^{k}, q^{k}\right) \rightarrow \varphi_{1}(x)=\left(r_{1}, \ldots, r_{n}, q\right)$. If for a given coordinate index $j$ the coordinate $r_{j}^{k}$ vanishes for all but finitely many $k$, then $r_{j}=0$, and if $r_{j}^{k}>0$ for all but finitely many $k$, then $r_{j} \geq 0$. Hence $d\left(x_{k}\right) \leq d(x)$, contradicting our assumption. The proof of Theorem 3.11 is complete.

Definition 3.14. The closure of a connected component of the set $X(1)=\{x \in X \mid$ $d(x)=1\}$ is called $a$ face of the M-polyfold $X$.

Around every point $x_{0} \in X$ there exists an open neighborhood $U=U\left(x_{0}\right)$ so that every $x \in U$ belongs to precisely $d(x)$ faces of $U$. This is easily verified. Globally it is always true that $x \in X$ belongs to at most $d(x)$ faces and the strict inequality is possible. 
Definition 3.15. The M-polyfold $X$ is called face structured if every point $x \in X$ belongs to precisely $d(x)$ faces.

This concept is related to some notion occurring in [15].

If $X \times Y$ is a product of two M-polyfolds, then one deduces from the definition of the product structure the following relation between the degeneracy indices:

$$
d_{X \times Y}(x, y)=d_{X}(x)+d_{Y}(y) .
$$

\subsection{Submanifolds}

There are many different types of distinguished subsets of an M-polyfold which qualify as some kind of sub-polyfold. We refer the reader to [6] for a comprehensive discussion, where we introduced three different notions of a sub-polyfold. Among those one can find sub-polyfolds of locally constant finite dimensions. These occur as solution sets of nonlinear Fredholm operators. In this paper we only consider the latter and introduce the notion of a strong submanifold of an M-polyfold. The more general notion of a submanifold requires some more work and is given in [8]. We just note that both types of submanifolds inherit from the ambient M-polyfold the structure of a smooth manifold. The strong submanifolds however lie in a better way in the M-polyfold.

We consider two sc-smooth splicings

$$
\mathcal{S}=(\pi, E, V) \quad \text { and } \quad \mathcal{T}=(\rho, F, V)
$$

having projections $\pi_{v}$ and $\rho_{v}$ parametrized by the same open subset $V$ of a partial quadrant. We define their Whitney sum to be the sc-smooth splicing

$$
\mathcal{S} \oplus \mathcal{T}=(\pi \oplus \rho, E \oplus F, V)
$$

defined by the family of projections

$$
(\pi \oplus \rho)_{v}(h \oplus k)=\left(\pi_{v}(h), \rho_{v}(k)\right), \quad v \in V .
$$

One verifies readily that the splicing core $K^{\mathcal{S} \oplus \mathcal{T}}$ is the fibered sum over $V$ of the splicing cores $K^{\mathcal{S}}$ and $K^{\mathcal{T}}$,

$$
\left.K^{\mathcal{S} \oplus \mathcal{T}}=K^{\mathcal{S}} \oplus_{V} K^{\mathcal{T}}=\{(v, h, k)) \in V \oplus E \oplus F \mid \pi_{v}(h)=h \text { and } \rho_{v}(k)=k\right\} .
$$

Definition 3.16. The sc-smooth map $f: X \rightarrow Y$ between two M-polyfolds is called $a$ fred-submersion if at every point $x_{0} \in X$ resp. $f\left(x_{0}\right) \in Y$ there exists a chart $(U, \varphi$, $\mathcal{T} \oplus \widehat{\mathcal{T}})$ resp. $(W, \psi, \mathcal{T})$ satisfying $f(U) \subset W$ and

$$
\psi \circ f \circ \varphi^{-1}\left(v, e^{\prime}, e^{\prime \prime}\right)=\left(v, e^{\prime}\right)
$$

and, moreover, the splicing $\widehat{\mathcal{T}}=(\widehat{\rho}, E, V)$ has the special property that the projections $\widehat{\rho}_{v}$ do not depend on $v$ and project onto a finite-dimensional subspace of $E$. 
Instead of $\widehat{\mathcal{T}}=(\widehat{\rho}, E, V)$ we may just take the splicing $\left(\mathrm{Id}, \mathbb{R}^{n}, V\right)$ where $n$ is the dimension of the image of the projection $\pi$ and Id stands for the constant family $v \rightarrow$ Id. Hence we may assume that in the Whitney sum $\mathcal{T} \oplus \widehat{\mathcal{T}}$ the latter summand has the special form and we will indicate that by writing

\section{$\mathcal{T} \oplus \mathbb{R}^{n}$}

The following result will be used quite often.

Proposition 3.17. If $f: X \rightarrow Y$ and $g: Y \rightarrow Z$ are fred-submersions, then the composition $g \circ f: X \rightarrow Z$ is again a fred-submersion.

Proof. Let $y_{0}=f\left(x_{0}\right)$ and $z_{0}=g\left(y_{0}\right)$. We find special charts $\phi$ and $\psi$ around $x_{0}$ and $y_{0}$, respectively, so that

$$
\psi \circ f \circ \phi^{-1}\left(v, e, e^{\prime}\right)=(v, e) .
$$

Similarly, we find special charts $\alpha$ and $\beta$ so that

$$
\alpha \circ g \circ \beta^{-1}\left(w, h, h^{\prime}\right)=(w, h) .
$$

Define the inverse of a chart $\gamma$ around $x_{0}$ by

$$
\gamma^{-1}\left(w, h, h^{\prime}, e^{\prime}\right)=\phi^{-1}\left(\psi \circ \beta^{-1}\left(w, h, h^{\prime}\right), e^{\prime}\right) .
$$

Then we compute

$$
\begin{aligned}
\alpha \circ(g \circ f) \circ \gamma^{-1}\left(w, h, h^{\prime}, e^{\prime}\right) & =\alpha \circ g \circ f \circ \phi^{-1}\left(\psi \circ \beta^{-1}\left(w, h, h^{\prime}\right), e^{\prime}\right) \\
& =\alpha \circ g \circ \psi^{-1} \circ\left(\psi \circ f \circ \phi^{-1}\right)\left(\psi \circ \beta^{-1}\left(w, h, h^{\prime}\right), e^{\prime}\right) \\
& =\alpha \circ g \circ \psi^{-1} \circ\left(\psi \circ \beta^{-1}\left(w, h, h^{\prime}\right)\right) \\
& =\alpha \circ g \circ \beta^{-1}\left(w, h, h^{\prime}\right)=(w, h) .
\end{aligned}
$$

The splicings used for the charts involved are of the form $\mathcal{S}$ and $\mathcal{S} \oplus\left(\mathbb{R}^{n} \oplus \mathbb{R}^{k}\right)$. This completes the proof.

The preimages of smooth points under a fred-submersion carry in a natural way the structure of smooth manifolds.

Proposition 3.18. If $f: X \rightarrow Y$ is a fred-submersion between two M-polyfolds, then the preimage of a smooth point $y \in Y$,

$$
f^{-1}(y) \subset X
$$

carries in a natural way the structure of a finite-dimensional smooth manifold.

Proof. We can define local charts induced from the charts of $X$ (exhibiting $f$ as a fredsubmersion). They are defined on open subsets in $\mathbb{R}^{n}$. Here $n$ is locally constant, i.e. only depends on the connected components of $X$. The transition maps are sc-smooth and consequently smooth in the classical sense. In other words, there is a natural system of charts which define the structure of a smooth manifold on $f^{-1}(y)$. 
The above discussion prompts the following useful concept.

Definition 3.19. A subset $N \subset X$ of an $M$-polyfold $X$ is called $a$ strong finite-dimensional submanifold of $X$ if the following statements hold true.

(i) $N \subset X_{\infty}$.

(ii) For every point $m \in N$ there exists an open neighborhood $U \subset X$ of $m$, an $M$ polyfold $Y$, and a surjective fred-submersion $f: U \rightarrow Y$ satisfying

$$
f^{-1}(f(m))=N \cap U .
$$

The definition of a finite-dimensional submanifold of an M-polyfold will be given in [8].

\section{M-polyfold bundles}

In this section we continue with the conceptual framework. First we describe the local models for strong M-polyfold bundles and smooth maps between them. Then we introduce the notion of a strong M-polyfold bundle.

\subsection{Local strong M-polyfold bundles}

In this subsection we shall introduce the local models for strong bundles over M-polyfolds. For this we need a generalization of the notion of splicing where the splicing projection is parameterized by an open subset of a splicing core. We begin by introducing these more general splicing definitions.

Definition 4.1. A general sc-smooth splicing is a triple

$$
\mathcal{R}=(\rho, F,(O, \mathcal{S})),
$$

where $(O, \mathcal{S})$ is a local M-polyfold model associated with the sc-smooth splicing $\mathcal{S}=(\pi, E, V)$ and $O$ is an open subset of the splicing core $K^{\mathcal{S}}=\{(v, e) \in V \oplus E \mid$ $\left.\pi_{v}(e)=e\right\}$. The space $F$ is an sc-smooth Banach space and the mapping

$$
\rho: O \oplus F \rightarrow F:((v, e), u) \mapsto \rho(v, e, u)
$$

is sc-smooth. Finally, for fixed $(v, e) \in O$, the mapping

$$
\rho_{(v, e)}=\rho(v, e, \cdot): F \rightarrow F
$$

is a projection in $\mathcal{L}(F)$. Sc-smoothness of $\rho$, of course, means that the map

$$
(v, e, u) \mapsto \rho\left(v, \pi_{v}(e), u\right),
$$

which is defined on an open subset $\widehat{O}$ of a partial quadrant in an sc-Banach space, is sc-smooth. 
The novelty of this definition consists in the requirement that the family of projections is parameterized by elements of an open subset of a splicing core. Iterating this procedure we obtain splicings parameterized by open sets in splicing cores of generalized splicings. Continuing this way we arrive at a hierarchy of splicings of the following types:

$$
\begin{gathered}
(v, e) \mapsto\left(v, \pi_{v}(e)\right), \\
(v, e, u) \mapsto\left(v, \pi_{v}(e), \rho_{\left(v, \pi_{v}(e)\right)}(u)\right), \\
(v, e, u, w) \mapsto\left(v, \pi_{v}(e), \rho_{\left(v, \pi_{v}(e)\right)}(u), \sigma_{\left(v, \pi_{v}(e), \rho_{\left(v, \pi_{v}(e)\right)}(u)\right)}(w)\right),
\end{gathered}
$$

and so on. Hence there are splicings of type 0 , which are the original ones, then there are splicings of type 1 , which are the generalized splicings introduced above, and so on. A type- $k$ splicing can also be viewed as a type $\ell$-splicing for every $\ell \geq k$. The notion of $\operatorname{sc}^{r}$ smoothness generalizes to these more general splicings. Using open sets of splicing core of splicings of type $k$ as local models we can construct $M$-polyfolds of type $k$ the same way we did in Definition 3.8 for the original M-polyfolds, which now become M-polyfolds of type 0 . In this paper we shall only meet M-polyfolds of type 0 and of type 1 .

The tangent of a general sc-smooth splicing $\mathcal{R}=(\rho, F,(O, \mathcal{S}))$ is defined, quite similarly to the case of a splicing, by

$$
T \mathcal{R}=(T \rho, T F,(T O, T \mathcal{S})),
$$

which is again a general sc-smooth splicing. The map $T \rho: T O \oplus T F \rightarrow T F$ is a family of projections acting on $T F$ and parameterized by the tangent $T O$ of $O$. It is defined by

$$
T \rho(w, \delta w, u, \delta u)=(\rho(w, u), D \rho(w, u)(\delta w, \delta u)) .
$$

Here $w=(v, e) \in O_{1} \subset V_{1} \oplus E_{1}$ and $\delta w \in W \oplus E$ so that $(w, \delta w) \in T O$ and $(u, \delta u) \in F^{1} \oplus F=T F$. Keeping $(w, \delta w) \in T O$ fixed, the map

$$
T \rho_{(w, \delta w)}: T F \rightarrow T F
$$

is a projection in $\mathcal{L}\left(F_{1} \oplus F\right)$.

Next we introduce the notion of a strong bundle splicing.

Definition 4.2. A strong bundle splicing is a general sc-smooth splicing

$$
\mathcal{R}=(\rho, F,(O, \mathcal{S}))
$$

having the following additional property. If $(v, e) \in O_{m}$ and $u \in F_{m+1}$, then $\rho((v, e), u)$ $\in F_{m+1}$ and the newly defined triple

$$
\mathcal{R}^{1}=\left(\rho, F^{1},(O, \mathcal{S})\right)
$$

is also a general sc-smooth splicing. If we view the strong bundle splicing $\mathcal{R}$ only as a general smooth splicing we denote it by $\mathcal{R}^{0}$. 
Let us note that the complementary splicing $\mathcal{R}^{c}$ is a strong bundle splicing as well. From the above definition we conclude, in particular, that a strong bundle splicing $\mathcal{R}$ gives rise to two general sc-smooth splicings, namely $\mathcal{R}^{0}$ and $\mathcal{R}^{1}$.

There is a nonsymmetric product $E \triangleleft F$ of two sc-Banach spaces $E$ and $F$. This product is the Banach space $E \oplus F$ equipped, however, with the bi-filtration defined by

$$
(E \triangleleft F)_{m, k}=E_{m} \oplus F_{k}
$$

for pairs $(m, k)$ satisfying $m \geq 0$ and $0 \leq k \leq m+1$. For a subset $U \subset E$ we can define $U \triangleleft F$ in the obvious way.

The splicing core $K^{\mathcal{R}}$ of the strong bundle splicing $\mathcal{R}=(\rho, F,(O, \mathcal{S}))$ is the set

$$
K^{\mathcal{R}}=\{(w, u) \in O \oplus F \mid \rho(w, u)=u\} .
$$

Since $\mathcal{R}$ gives us two general splicings $\mathcal{R}^{0}$ and $\mathcal{R}^{1}$ we have a well defined bi-filtration on $K^{\mathcal{R}}$ by pairs $(m, k)$ satisfying $0 \leq k \leq m+1$ so that $K^{\mathcal{R}}$ can be viewed as a subset of $(V \oplus E) \triangleleft F$ equipped with the induced bi-filtration. More precisely,

$$
K_{m, k}^{\mathcal{R}}=\left\{(w, u) \in K^{\mathcal{R}} \mid w \in O_{m}, u \in F_{k}\right\}
$$

where $m \geq 0$ and $0 \leq k \leq m+1$. The bundle

$$
K^{\mathcal{R}} \rightarrow O
$$

defined by means of the strong bundle splicing $\mathcal{R}$ is called a local strong bundle. It will serve as our local model of the strong M-polyfold bundles introduced in the next subsection.

Remark 4.3. There is a special case which we already briefly mentioned before. Assume the strong bundle splicing $\mathcal{R}$ has the special form

$$
\mathcal{R}=(\operatorname{Id}, F,(O, \mathcal{S})),
$$

where $\mathcal{S}=(\mathrm{Id}, E, V)$ and $O$ is a relatively open subset of $V \oplus E$. In this case the splicing core is the product

$$
K^{\mathcal{R}}=O \triangleleft F
$$

and we can view $O$ as a local model for an sc-manifold and the product $O \triangleleft F$ as a model for a local strong sc-bundle with base $O$.

Associated with the strong bundle splicing $\mathcal{R}$ we have the splicing cores $K^{\mathcal{R}^{0}}$ and $K^{\mathcal{R}^{1}}$, which we denote by $K^{\mathcal{R}}(0)$ and $K^{\mathcal{R}}(1)$, respectively. They are equipped with the filtrations

$$
K^{\mathcal{R}}(0)_{m}=K_{m, m}^{\mathcal{R}} \quad \text { and } \quad K^{\mathcal{R}}(1)_{m}=K_{m, m+1}^{\mathcal{R}} .
$$

The natural projection $K^{\mathcal{R}} \rightarrow O:(w, u) \mapsto u$ is sc-smooth in the sense that the two projections $K^{\mathcal{R}}(i) \rightarrow O$ are sc-smooth for $i=0,1$.

We can define the tangent $T \mathcal{R}$ of the strong bundle splicing

$$
\mathcal{R}=(\rho, F,(O, \mathcal{S}))
$$


as follows. First we consider the underlying strong bundle splicing $\mathcal{R}^{0}$ and take the associated tangent splicing $T \mathcal{R}^{0}$,

$$
T \mathcal{R}^{0}=(T \rho, T F,(T O, T \mathcal{S}))
$$

Since we also have the splicing $\mathcal{R}^{1}$, we can take its tangent $T \mathcal{R}^{1}$ given by

$$
T \mathcal{R}^{1}=\left(T \rho, T\left(F^{1}\right),(T O, T \mathcal{S})\right) .
$$

From $T\left(F^{1}\right)=(T F)^{1}$ we conclude that

$$
T \mathcal{R}=(T \rho, T F,(T O, T \mathcal{S}))
$$

is again a strong bundle splicing in the sense of Definition 4.2 . Its splicing core $K^{T \mathcal{R}}$ is, as usual, defined by

$$
K^{T \mathcal{R}}=\{(w, \delta w, u, \delta u) \in T O \oplus T F \mid T \rho(w, \delta w, u, \delta u)=(u, \delta u)\} .
$$

More explicitly, the elements of $K^{T \mathcal{R}}$ are restricted by the following equations for $w=$ $(v, e) \in O_{1} \oplus E_{1}$ and $\delta w=(\delta v, \delta e) \in W \oplus E$ so that $(w, \delta w) \in T O$ and for $(u, \delta u)$ $\in T F$ :

$$
\begin{aligned}
\pi(v, e)=e, & \delta e=\pi(v, \delta e)+D_{v} \pi(v, e) \delta v, \\
\rho(w, u)=u, & \delta u=\rho(w, \delta u)+D_{w} \rho(w, u) \delta w .
\end{aligned}
$$

Let us observe that for $i=0,1$ the following relationships hold for the underlying general sc-smooth splicings:

$$
\left(T K^{\mathcal{R}}\right)(i)=K^{T \mathcal{R}}(i)=K^{T\left(\mathcal{R}^{i}\right)}=T\left(K^{\mathcal{R}}(i)\right) .
$$

Next we shall define the concept of a strong bundle map of class $\mathrm{sc}_{\triangleleft}^{k}$ between splicing cores of strong bundle splicings. Recall Definition 3.4 for the $\mathrm{sc}^{1}$-class of mappings between open subsets of splicing cores.

Definition 4.4. If $\mathcal{R}=(\rho, F,(O, \mathcal{S}))$ and $\mathcal{R}^{\prime}=\left(\rho^{\prime}, F^{\prime},\left(O^{\prime}, \mathcal{S}^{\prime}\right)\right)$ are two strong bundle splicings we denote the associated splicing cores by $K=K^{\mathcal{R}} \subset O \oplus F$ and $K^{\prime}=K^{\mathcal{R}^{\prime}} \subset$ $O^{\prime} \oplus F^{\prime}$. Consider a map $f: K \rightarrow K^{\prime}$ of the form

$$
f(w, u)=(\varphi(w), \Phi(w, u)),
$$

where $\varphi: O \rightarrow O^{\prime}$ and $\Phi: O \oplus F \rightarrow F^{\prime}$.

- The map $f$ is a strong bundle map of class $\mathrm{sc}_{\triangleleft}^{0}$, or simply an $s c_{\triangleleft}^{0}$-map, if it induces $s c^{0}$-maps $K(i) \rightarrow K^{\prime}(i)$ for $i=0$ and $i=1$.

- The map $f$ is a strong bundle map of class $\mathrm{sc}_{\triangleleft}^{1}$ if it is of class $s c_{\triangleleft}^{0}$ and if it induces $s c^{1}$-maps $K(i) \rightarrow K^{\prime}(i)$ for $i=0$ and $i=1$.

Observe that $K(i)$ and $K^{\prime}(i)$ are type-1 M-polyfolds. 
In many cases we require $\Phi$ to be linear in $u$. In particular, this is the case when $\Phi$ occurs as an isomorphism between local strong M-polyfold bundles.

Next we consider maps $f: K \rightarrow K^{\prime}$ between splicing cores of strong bundle splicings of the form as in Definition 4.4 In order to define maps $f: K \rightarrow K^{\prime}$ of class $\mathrm{sc}_{\triangleleft}^{2}$ we proceed as in the sc-case. Assuming that $f$ is of class $\operatorname{sc}_{\triangleleft}^{1}$ we consider it first as an $\mathrm{sc}^{1}$-map

$$
f: K(0) \rightarrow K^{\prime}(0)
$$

between splicing cores. Its tangent map $T f$ is described by the formula

$$
T f(w, \delta w, u, \delta u)=(\varphi(w), D \varphi(w) \delta w, \Phi(w, u), D \Phi(u, w)(\delta w, \delta u)) .
$$

Since $f$ is also an $\mathrm{sc}^{1}$-map

$$
f: K(1) \rightarrow K^{\prime}(1)
$$

the tangent formula above defines two maps

$$
T f: T K^{\mathcal{R}}(i)=K^{T \mathcal{R}^{i}} \rightarrow T K^{\mathcal{R}^{\prime}}(i)=K^{T \mathcal{R}^{\prime i}}
$$

for $i=0,1$ which are $\mathrm{sc}^{0}$-continuous. Therefore they define a map

$$
T f: T K^{\mathcal{R}} \rightarrow T K^{\mathcal{R}^{\prime}}
$$

between splicing cores of strong bundle splicings which is of class $\operatorname{sc}_{\triangleleft}^{0}$. It is called the tangent map of the $\mathrm{sc}_{\triangleleft}^{1}$-map $f$.

If this tangent map $T f$ is of class $\operatorname{sc}_{\triangleleft}^{1}$ as defined above, then the map $f: K \rightarrow K^{\prime}$ is called of class $s c_{\triangleleft}^{2}$. Proceeding inductively as in the sc-case one defines the mappings $f: K \rightarrow K^{\prime}$ of class $\mathrm{sc}_{\triangleleft}^{k}$ for $k \geq 1$ and also the $\mathrm{sc}_{\triangleleft}$-smooth mappings. Let us finally note that the chain rule also holds for strong bundle maps.

Theorem 4.5 (Chain rule for strong bundle maps). Let $f: K \mid O \rightarrow K^{\prime}$ and $g: K^{\prime} \mid O^{\prime}$ $\rightarrow K^{\prime \prime}$ be two strong bundle maps of class $s c_{\triangleleft}^{1}$ between local strong bundles so that the image of $f$ is contained in the domain of $g$. Then the composition $g \circ f$ is also a strong bundle map of class $s c_{\triangleleft}^{1}$ and the tangent maps satisfy

$$
T(g \circ f)=T g \circ T f \text {. }
$$

A note of caution: as before the order of terms in the tangent map $T f$ of an $\mathrm{sc}_{\triangleleft}^{1}$-map is different from their order in the classical theory.

Associated with the strong bundle splicing $\mathcal{R}$ we have the local strong bundle $p$ : $K \rightarrow O$. An sc-smooth section of the bundle $p$ is just an sc-smooth section of the underlying bundle $K(0) \rightarrow O$. The vector space of sc-smooth sections is denoted by $\Gamma(p)$. In addition, there is a different class of sections called sc ${ }^{+}$-sections. An sc-smooth section $f$ is called an $s c^{+}$-section if it defines an sc-smooth section of the bundle $K(1) \rightarrow O$. We denote the collection of $\mathrm{sc}^{+}$-sections by $\Gamma^{+}(p)$. 


\subsection{Fillability and fillers}

Considering the local strong bundle $p: K^{\mathcal{R}} \rightarrow O$ associated with a strong bundle splicing $\mathcal{R}$, we investigate the coherence in the jumps of the space dimensions in the base and the fibers.

We start with a strong bundle splicing $\mathcal{R}=(\rho, F,(O, \mathcal{S}))$. The splicing $\mathcal{S}$ is the triple $(\pi, E, V)$ in which $V$ is an open subset of a partial quadrant $C$ contained in the sc-Banach space $W$. The set $O$ is an open neighborhood of the origin in the splicing core $K^{\mathcal{S}}=\left\{(v, e) \in V \oplus E \mid \pi_{v}(e)=e\right\}$. If

$$
s: O \rightarrow V
$$

is the sc-smooth map defined by $s(v, e)=v$, we shall abbreviate by $s^{*} \pi: O \times E \rightarrow E$ the composition $s^{*} \pi(v, e, u)=\pi(s(v, e), u)=\pi(v, u)$ and introduce the general sc-smooth splicing $s^{*} \mathcal{S}^{c}$, having the splicing parameter set $O$, by

$$
s^{*} \mathcal{S}^{c}=\left(1-s^{*} \pi, E, O\right) .
$$

Its splicing core is the set

$$
\begin{aligned}
K^{s^{*} \mathcal{S}^{c}} & =\left\{(v, e, u) \in O \oplus E \mid \pi_{s(v, e)}(u)=0\right\} \\
& =\left\{(v, e, u) \in V \oplus E \oplus E \mid(v, e) \in O, \pi_{v}(e)=e \text { and }\left(1-\pi_{v}\right)(u)=u\right\} .
\end{aligned}
$$

In view of the splitting $E=\pi_{v}(E) \oplus\left(1-\pi_{v}\right)(E)$, the splicing core $K^{s^{*} \mathcal{S}^{c}}$ can be naturally identified with the following open subset $\widehat{O}$ of $V \oplus E$ :

$$
\widehat{O}=\left\{(v, e) \in V \oplus E \mid\left(v, \pi_{v}(e)\right) \in O\right\} .
$$

We have the natural projection

$$
a: K^{s^{*} \mathcal{S}^{c}} \rightarrow O:(v, w) \mapsto\left(v, \pi_{v}(w)\right)
$$

and we can view $a: K^{s^{*} \mathcal{S}^{c}} \rightarrow O$ as a bundle (of course, not as a strong bundle). The fiber $a^{-1}(v, e)$ over the point $(v, e) \in O$ is the Banach space

$$
a^{-1}(v, e)=\left\{(v, e+w) \mid w \in E, \pi_{v}(w)=0\right\}=\{(v, e)\} \times \operatorname{ker}\left(\pi_{v}\right) .
$$

The strong bundle splicing $\mathcal{R}$ comes together with its complementary strong bundle splicing $\mathcal{R}^{c}=(1-\rho, F,(O, \mathcal{S}))$ giving rise to the local strong bundle $b: K^{\mathcal{R}^{c}} \rightarrow O$. We are interested only in the underlying bundle

$$
b: K^{\mathcal{R}^{c}}(0) \rightarrow O
$$

associated with the general sc-smooth splicing $\left(\mathcal{R}^{c}\right)^{0}$.

The following concept of a filler turns out to be very useful in the applications. 
Definition 4.6. Let $\mathcal{R}$ be a strong bundle splicing and $\mathcal{R}^{c}$ the associated complementary strong bundle splicing. Consider the two bundles over $O$,

$$
a: K^{s^{*} \mathcal{S}^{c}} \rightarrow O \text { and } b: K^{\mathcal{R}^{c}}(0) \rightarrow O .
$$

Then a filler for $\mathcal{R}$ is an sc-diffeomorphism

$$
f^{c}: K^{s^{*} \mathcal{S}^{c}} \rightarrow K^{\mathcal{R}^{c}}(0)
$$

between the complementary bundle pairs, which is linear in the fibers and covers the identity map $O \rightarrow O$. (It is, in particular, a bundle isomorphism.)

Definition 4.7. The strong bundle splicing $\mathcal{R}$ is fillable if there exists a filler for $\mathcal{R}$.

Being fillable is a property of the strong bundle splicing $\mathcal{R}$.

A filler $f^{c}: K^{s^{*} \mathcal{S}^{c}} \rightarrow K^{\mathcal{R}^{c}}(0)$ has the the form

$$
f^{c}:(v, e, u) \mapsto\left(v, e, \mathbf{f}^{c}(v, e, u)\right)
$$

where $(v, e) \in O$ and where $u \in E$ satisfies $\pi_{v}(u)=0$. The principal part $\mathbf{f}^{c}(v, e, u) \in F$ satisfies $\rho_{(v, e)}\left(\mathbf{f}^{c}(v, e, u)\right)=0$. In view of the identity $e=\pi_{v}(e)+\left(1-\pi_{v}\right)(e)$ in $E$, the principal part $\mathbf{f}^{c}$ can be viewed as an sc-smooth map

$$
\widehat{O} \rightarrow F:(v, e) \mapsto \mathbf{f}^{c}(v, e)
$$

satisfying $\rho_{\left(v, \pi_{v}(e)\right)}\left(\mathbf{f}^{c}(v, e)\right)=0$.

\subsection{Strong M-polyfold bundles}

In order to introduce strong M-polyfold bundles we consider a surjective sc-smooth map $p: Y \rightarrow X$ between two M-polyfolds. The M-polyfold $Y$ is of type-1 and $X$ of type- 0 . We assume in addition that for every $x \in X$, the preimage $p^{-1}(x)=Y_{x}$, called the fiber over $x$, carries the structure of a Banach space.

Definition 4.8. Let $p: Y \rightarrow X$ be as just described. A strong M-polyfold bundle chart for the bundle $p: Y \rightarrow X$ is a triple $\left(U, \Phi,\left(K^{\mathcal{R}}, \mathcal{R}\right)\right)$. Here $U \subset X$ is an open set and $\mathcal{R}=(\rho, F,(O, \mathcal{S}))$ a strong bundle splicing with the local model $(O, \mathcal{S})$ of the polyfold $X$. The map $\Phi$ is an sc-diffeomorphism

$$
\Phi: p^{-1}(U) \rightarrow K^{\mathcal{R}}
$$

which is linear on the fibers and covers the sc-diffeomorphism

$$
\varphi: U \rightarrow O
$$

so that $\mathrm{pr}_{1} \circ \Phi=\varphi \circ p$. Moreover, the maps $\Phi$ resp. $\varphi$ are smoothly compatible with the $M$-polyfold structures on $Y$ and $X$, respectively.

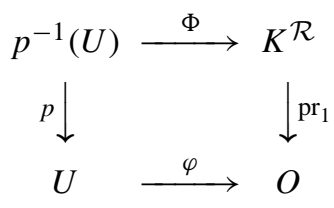


Recall that $\mathcal{S}=(\pi, E, V)$ is an sc-smooth splicing where $V$ is an open subset of a partial quadrant in an sc-smooth Banach space $W$. The set $O$ is an open subset of the splicing core $K^{\mathcal{S}}=\{w=(v, e) \in V \oplus E \mid \pi(v, e)=e\}$ while the splicing core $K^{\mathcal{R}}$ over the base $O$ is defined by $K^{\mathcal{R}}=\{(w, u) \in O \oplus F \mid \rho(w, u)=u\}$ where $F$ is an sc-smooth Banach space.

Definition 4.9. Two M-polybundle charts $(\Phi, \varphi)$ and $(\Psi, \psi)$ are called $\mathrm{sc}_{\triangleleft}$-compatible if the transition map

$$
\Psi \circ \Phi^{-1}: K^{\mathcal{R}}\left|\varphi\left(U \cap U^{\prime}\right) \rightarrow K^{\mathcal{R}^{\prime}}\right| \psi\left(U \cap U^{\prime}\right)
$$

between their splicing cores $K^{\mathcal{R}}$ and $K^{\mathcal{R}^{\prime}}$ is an $s c_{\triangleleft}$-smooth strong bundle map.

An M-polybundle atlas consists of a family of M-polybundle charts $\left(U, \Phi,\left(K^{\mathcal{R}}, \mathcal{R}\right)\right)$ so that the underlying open sets $U$ cover $X$ and the transition maps are $\mathrm{SC}_{\triangleleft}$-smooth strong bundle maps. A maximal smooth atlas of M-polybundle charts is called an M-polybundle structure and the bundle $p: Y \rightarrow X$ is called a strong M-polyfold bundle.

Definition 4.10. A strong $M$-polyfold bundle $p: Y \rightarrow X$ is called fillable if around every point $q \in X$, there exists a compatible strong M-polyfold bundle chart $\left(U, \Phi,\left(K^{\mathcal{R}}, \mathcal{R}\right)\right)$ whose strong bundle splicing $\mathcal{R}$ is fillable.

It turns out that all strong bundles occurring in the applications we have in mind have this property.

Note that in general the tangent bundle $T X \rightarrow X^{1}$ is not a strong M-polyfold bundle.

Given the strong polyfold bundle $p: Z \rightarrow X$ having the base $X$ and the sc-smooth map $f: Y \rightarrow X$ between M-polyfolds one defines the (algebraic) pullback (or induced) bundle

$$
p^{\prime}: f^{*} Z \rightarrow Y
$$

having the base $Y$ as follows. One takes the set $f^{*} Z=\{(y, z) \in Y \times Z \mid p(z)=f(y)\}$ and the two projection maps $p^{\prime}(y, z)=y$ and $f^{\prime}(y, z)=z$, so that the diagram

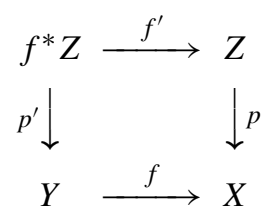

commutes.

Proposition 4.11. If $p: Z \rightarrow X$ is a strong $M$-polyfold bundle and $f: Y \rightarrow X$ is an sc-smooth map between M-polyfolds, then the pullback bundle $p^{\prime}: f^{*} Z \rightarrow Y$ carries a natural induced structure of a strong M-polyfold bundle whose base is the M-polyfold $Y$.

Proof. Choose a point $\left(y_{0}, z_{0}\right) \in f^{*} Z$ so that $f\left(y_{0}\right)=p\left(z_{0}\right)=x_{0} \in X$. Take a strong M-polyfold bundle chart for $p: Z \rightarrow X$ denoted by $\left(U, \Phi,\left(K^{\mathcal{R}}, \mathcal{R}\right)\right)$,

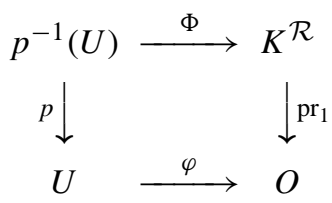


so that the open set $U \subset X$ contains the point $x_{0}$. The strong bundle splicing $\mathcal{R}=$ $(\rho, F,(O, \mathcal{S}))$ is associated with the local model $(O, \mathcal{S})$ of the polyfold $X$, where $O$ is an open subset of the splicing core $K^{\mathcal{S}}$ of the splicing $\mathcal{S}=(\pi, E, V)$. Take now an M-polyfold chart $\psi: U^{\prime} \rightarrow O^{\prime}$ around the given point $y_{0} \in U^{\prime} \in Y$, associated with the local model $\left(O^{\prime}, \mathcal{S}^{\prime}\right)$ of the M-polyfold $Y$, where $O^{\prime} \subset K^{\mathcal{S}^{\prime}}$ is an open subset of the splicing core of the sc-smooth splicing $\mathcal{S}^{\prime}=\left(\pi^{\prime}, E^{\prime}, V^{\prime}\right)$. Choose $U^{\prime} \subset Y$ so small that $f\left(U^{\prime}\right) \subset U$. Define the strong bundle splicing $\mathcal{R}^{\prime}=\left(\rho^{\prime}, F,\left(O^{\prime}, \mathcal{S}^{\prime}\right)\right)$ by means of the sc-smooth map $\rho^{\prime}: O^{\prime} \oplus F \rightarrow F$ given as

$$
\rho^{\prime}\left(v^{\prime}, u\right):=\rho\left(\varphi \circ f \circ \psi^{-1}\left(v^{\prime}\right), u\right) .
$$

The strong M-polyfold bundle chart for the bundle $p^{\prime}: f^{*} Z \rightarrow Y$ is now the triple $\left(U^{\prime}, \Psi,\left(K^{\mathcal{R}^{\prime}}, \mathcal{R}^{\prime}\right)\right)$ with the homeomorphism

$$
\Psi: f^{*} Z\left|U^{\prime} \rightarrow K^{\mathcal{R}^{\prime}}\right| O^{\prime}
$$

defined as

$$
\Psi\left(p^{\prime-1}(y)\right)=\Phi\left(p^{-1}(f(y))\right)
$$

for all $y \in U^{\prime} \subset Y$. We have the diagram

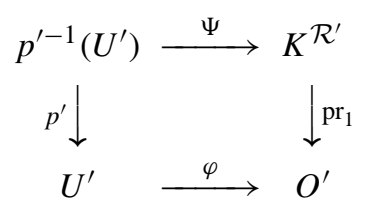

One can verify that the transition maps between two such strong M-polyfold bundle charts are $\mathrm{sc}_{\triangleleft}$-smooth. This finishes the proof of Proposition 4.11

\subsection{Sections and linearizations}

Assume that $p: Y \rightarrow X$ is a strong M-polyfold bundle over the M-polyfold $X$. We denote the space of sc-smooth sections by $\Gamma(p)$. In addition there is the distinguished space of $\mathrm{sc}^{+}$-sections which we denote by $\Gamma^{+}(p)$.

The section $f$ is an $s c^{+}$-section if its local representations in the strong Mpolyfold bundle charts are $\mathrm{sc}^{+}$-sections as defined at the end of Section 4.1 above. If $\left(U, \Phi,\left(K^{\mathcal{R}}, \mathcal{R}\right)\right)$ is such a strong M-polyfold bundle chart, the local representation of the section $f$ of the bundle $p: Y \rightarrow X$ is the section $g$ of the strong local bundle $K^{\mathcal{R}} \rightarrow O$ defined as the push-forward of $f$ by

$$
g(w)=\Phi \circ f \circ \varphi^{-1}(w),
$$

where $w \in O$. By definition, the map $\Phi: p^{-1}(U) \rightarrow K^{\mathcal{R}}$ is an sc-diffeomorphism which is linear in the fibers and which covers the sc-diffeomorphism $\varphi: U \rightarrow O$ where $O$ is an open subset of the splicing core $K^{\mathcal{S}}=\left\{(v, e) \mid \pi_{v}(e)=e\right\}$ belonging to the splicing $\mathcal{S}=(\pi, E, V)$. Associated with the strong bundle splicing $\mathcal{R}=(\rho, F,(O, \mathcal{S}))$ we have the splicing core $K^{\mathcal{R}}=\{(w, u) \in O \oplus F \mid \rho(w, u)=u\}$. 
Next we choose a smooth point $q \in X$. Generalizing a trivial classical fact for vector bundles we can identify naturally the tangent space $T_{0_{q}} Y$ at the zero element $0_{q}=\Phi^{-1}\left(\varphi(q), 0_{F}\right)$ with the sc-Banach space $T_{q} X \oplus Y_{q}$ where $Y_{q}=p^{-1}(q)$ is the fiber. Since $q$ is a smooth point we may assume in the following that $\varphi(q) \in O$ is equal to 0 , so that $\Phi\left(0_{q}\right)=(0,0) \in K^{\mathcal{R}} \subset O \oplus F$. The identification $T_{0_{q}} Y \leftrightarrow T_{q} X \oplus Y_{q}$ corresponds in the local coordinates to the identification

$$
(0, \delta w, 0, \delta u) \leftrightarrow((0, \delta w),(0, \delta u))
$$

of the elements in the tangent space $T_{(0,0)} K^{\mathcal{R}} \subset T_{0} O \oplus T_{0} F$. We shall denote by $P_{q}$ : $T_{0_{q}} Y \simeq T_{q} X \oplus Y_{q} \rightarrow Y_{q}$ the projection.

Given a section $f \in \Gamma(p)$ which vanishes at the smooth point $q \in X$ we define, following the classical recipe, the linearization $f^{\prime}(q)$ by

$$
f^{\prime}(q): T_{q} X \rightarrow Y_{q}: h \mapsto P_{q} \circ T f(q) h .
$$

As in the case of vector bundles there is generally no intrinsic notion of a linearization of a section at an arbitrary point $q$ at which $f(q)$ does not vanish. In our case with $Y \rightarrow X$ being a strong bundle we have, however, some additional structure. This will allow us to define a linearization at an arbitrary smooth point which is unique up to a linear $\mathrm{sc}^{+}$. operator.

In order to do this, we consider the sc-smooth section $f \in \Gamma(p)$ and look at the smooth point $q \in X$. Its image $y=f(q)$ is a smooth point in $Y$ and we claim that there exists an $\mathrm{sc}^{+}$-section $s$ defined near $q$ and satisfying $s(q)=f(q)$.

Indeed, if the coordinate representation of the section $f$ is given by $g(w)=(w, \mathbf{g}(w))$ and if $q$ corresponds to $w_{0}$, then $\mathbf{g}\left(w_{0}\right)$ is a smooth point in the sc-Banach space $F$ satisfying $\rho\left(w_{0}, \mathbf{g}\left(w_{0}\right)\right)=\mathbf{g}\left(w_{0}\right)$. Now define in the local coordinates the section $s$ by $s(w)=\left(w, \rho\left(w, \mathbf{g}\left(w_{0}\right)\right)\right)$. It satisfies $s\left(w_{0}\right)=\mathbf{g}\left(w_{0}\right)$ and is indeed an sc ${ }^{+}$-section because the projections $\rho$ belong to a strong bundle splicing as defined in Definition 4.2.

Now take any $\mathrm{sc}^{+}$-section $s$ of the bundle $Y \rightarrow X$ defined near $q$ and satisfying $s(q)=f(q)$. Then the section $f-s$ is defined near $q$ and vanishes at $q$. We define the linearization $f_{s}^{\prime}(q)$ by

$$
f_{s}^{\prime}(q): T_{q} X \rightarrow Y_{q}: h \mapsto P_{q} \circ T(f-s)(q) h .
$$

Next we investigate to what extent $f_{s}^{\prime}(q)$ depends on the choice of $s$. Assume therefore that $s$ and $t$ are $\operatorname{sc}^{+}$-sections defined near $q$ and satisfying $s(q)=t(q)=f(q)$. Then, by definition,

$$
\begin{aligned}
f_{s}^{\prime}(q) & =P_{q} \circ T(f-s)(q)=P_{q} \circ T(f-t+(t-s))(q) \\
& =P_{q} \circ T(f-t)(q)+P_{q} \circ T(t-s)(q)=f_{t}^{\prime}(q)+P_{q} \circ T(t-s)(q) .
\end{aligned}
$$

It remains to understand the perturbation term $P_{q} \circ T(t-s)(q)$. For this we observe that it suffices to understand $P_{q} \circ T s(q)$ for an $\operatorname{sc}^{+}$-section $s$ defined in a neighborhood $U \subset X$ of $q$ and vanishing at $q$. Since $s$ is an $\operatorname{sc}^{+}$-section of the bundle $p: Y \mid U \rightarrow U$, its tangent $T s$ is an $\mathrm{sc}^{+}$-section of $T p: T(Y \mid U) \rightarrow T U$. Hence the composition

$$
P_{q} \circ T s(q): T_{q} X \rightarrow Y_{q},
$$


which in local coordinates is given by

$$
(0, \delta w) \mapsto(0, \delta w, 0, D s(0) \delta w) \mapsto(0, D s(0) \delta w),
$$

is an $\mathrm{sc}^{+}$-operator.

Definition 4.12. Let $[f, q]$ be the germ of a section $f$ of the strong bundle $p: Y \rightarrow X$ around the smooth point $q$. Let $[s]$ be the germ of an $s^{+}$-section around $q$ which satisfies $s(q)=f(q)$. Then the linearization of $[f, q]$ with respect to $[s]$ is defined by

$$
f_{[s]}^{\prime}(q)=P_{q} \circ T(f-s)(q) .
$$

The above discussion is now summarized in the following proposition.

Proposition 4.13. Let $[f, q]$ be an sc-smooth section germ of the bundle $p: Y \rightarrow X$ near a smooth point $q$. Then two linearizations $f_{[s]}^{\prime}(q)$ and $f_{[t]}^{\prime}(q)$ differ by an $s c^{+}$-operator. In particular, if one linearization is sc-Fredholm so are all others.

The last statement follows from Proposition 2.11 This allows us to introduce the following definition.

Definition 4.14. An sc-smooth section of the strong M-polyfold bundle $p: Y \rightarrow X$ is linearized Fredholm at the smooth point $q$ provided a linearization at the point $q$ is scFredholm. We say $f$ is linearized Fredholm if this holds at all smooth points $q$.

If $f$ is linearized Fredholm and $q$ a smooth point, we define the index $\operatorname{Ind}(f, q) \in \mathbb{Z}$ by

$$
\operatorname{Ind}(f, q):=i\left(f_{[s]}^{\prime}(q)\right) .
$$

In view of Proposition 4.13 this is well defined. Here $i$ denotes the Fredholm index.

Another consequence of the previous discussion is the following.

Proposition 4.15. Assume that $p: Y \rightarrow X$ is a strong M-polyfold bundle and $f \in \Gamma(p)$ an sc-smooth section which is linearized Fredholm. Then the section $f+s$ for any sc ${ }^{+}$section in $\Gamma^{+}(p)$ is linearized Fredholm.

If a strong M-polyfold bundle $p: Y \rightarrow X$ is fillable one can construct for every scsmooth section $f$ near a smooth point $q \in X$ a filled section. This will be important for the Fredholm theory developed in [8]. To carry out this construction we assume that $f$ is an sc-smooth section of the bundle $p: Y \rightarrow X$ and $q \in X_{\infty}$. We pick a fillable strong bundle coordinate

$$
\Phi: Y \mid U \rightarrow K^{\mathcal{R}}
$$

defined on an open neighborhood $U$ of $q$ and covering the sc-diffeomorphism $\varphi: U \rightarrow O$. Let

$$
f^{c}: K^{s^{*} \mathcal{S}^{c}} \rightarrow K^{\mathcal{R}^{c}}(0)
$$

be a filler for the strong bundle splicing $\mathcal{R}=(\rho, F,(O,(\pi, E, V)))$. The principal part of $f^{c}$ gives us an sc-smooth map

$$
\mathbf{f}^{c}: \widehat{O} \rightarrow F:(v, e) \mapsto \mathbf{f}^{c}(v, e)
$$

satisfying $\rho_{\left(v, \pi_{v}(e)\right)}\left(\mathbf{f}^{c}(v, e)\right)=0$. Recall that $\widehat{O}$ stands for the open subset of $V \oplus E$ defined by $\widehat{O}=\left\{(v, e) \in V \oplus E \mid\left(v, \pi_{v}(e)\right) \in O\right\}$. The push-forward $\Phi_{*} f$ is a section 
of the local strong bundle $K^{\mathcal{R}} \rightarrow O$. Its principal part has a natural extension to the open set $\widehat{O}$ which we denote by f. It satisfies

$$
\rho_{\left(v, \pi_{v}(e)\right)}(\mathbf{f}(v, e))=\mathbf{f}(v, e) .
$$

Finally, we introduce the sc-smoth map $\overline{\mathbf{f}}: \widehat{O} \rightarrow F$ by

$$
\overline{\mathbf{f}}(v, e)=\mathbf{f}(v, e)+\mathbf{f}^{c}(v, e),
$$

which can be viewed as the principal part of an sc-smooth section $\bar{f}$ of the bundle $\widehat{O} \triangleleft F$ $\rightarrow \widehat{O}$.

Definition 4.16. The section $\bar{f}$ is called $a$ filled version of $f$ near $q$ and $\overline{\mathbf{f}}$ is called its principal part, i.e.

$$
\bar{f}(v, e)=((v, e), \overline{\mathbf{f}}(v, e)) .
$$

Let us observe that $\bar{f}(v, e)=0$ if and only if $\mathbf{f}(v, e)=0$ and $\mathbf{f}^{c}(v, e)=0$. Since $f^{c}$ is a filler we deduce from $\mathbf{f}^{c}(v, e)=0$ that $\left(1-\pi_{v}\right)(e)=0$, implying $\pi_{v}(e)=e$ so that $(v, e) \in O$ and $\Phi_{*} f(v, e)=0$. Hence $\varphi^{-1}(v, e)$ is a zero of the section $f$. Consequently, a filled version still describes in local coordinates precisely the solution set of $f$ over the set $U$.

Assume again that $p: Y \rightarrow X$ is a fillable strong M-polyfold bundle and $f$ an sc-smooth section. Suppose that $\bar{f}$ is a filled version representing the section $f \mid U$ as a section of the bundle $\widehat{O} \triangleleft F \rightarrow \widehat{O}$. We will prove the following result.

Proposition 4.17. For a smooth point $q \in U$ corresponding to the point $(v, e) \in O$ the linearization $f_{[s]}^{\prime}(q): T_{q} X \rightarrow Y_{q}$ is sc-Fredholm if and only if the linearization of the filled version $\bar{f}_{[t]}^{\prime}(v, e): T_{(v, e)} \widehat{O} \rightarrow F$ is sc-Fredholm. In this case the Fredholm indices are the same.

Proof. Without loss of generality we may assume that $f(q)=0$ and $s=0$. Using fillable strong bundle coordinates $\Phi: Y \mid U \rightarrow K^{\mathcal{R}}$ covering $\varphi: U \rightarrow O$ we may assume without loss of generality that $f$ is an sc-smooth section of the bundle

$$
p: K^{\mathcal{R}} \rightarrow O
$$

satisfying $0 \in O$ and $f(0)=0$. Then $f$ has the form

$$
O \rightarrow K^{\mathcal{R}}:(v, e) \mapsto((v, e), \widehat{f}(v, e)) .
$$

Using its principal part $\widehat{f}$ we can define an sc-smooth map

$$
\mathbf{f}: \widehat{O} \rightarrow F:(v, e) \mapsto \widehat{f}\left(v, \pi_{v}(e)\right),
$$

which satisfies $\rho_{\left(v, \pi_{v}(e)\right)}(\mathbf{f}(v, e))=\mathbf{f}(v, e)$. The open subset $\widehat{O}$ of $V \oplus E$ can be naturally identified with the splicing core $K^{s^{*} \mathcal{S}^{c}}$, as we have seen in Section 4.2 Since the strong bundle splicing $\mathcal{R}$ is fillable, we have the existence of a bundle isomorphism (linear in the fibers)

$$
K^{s^{*} \mathcal{S}^{c}} \rightarrow K^{\mathcal{R}^{c}}(0)
$$

over the set $O$ which gives us the sc-smooth filler

$$
\mathbf{f}^{c}: \widehat{O} \rightarrow F
$$


satisfying $\rho_{\left(v, \pi_{v}(e)\right)}\left(\mathbf{f}^{c}(v, e)\right)=0$. In addition, for fixed $(v, e) \in O$ the map $r \mapsto$ $\mathbf{f}^{c}(v, e+r)$ is a linear isomorphism between the $\operatorname{Banach} \operatorname{spaces} \operatorname{ker}\left(\pi_{v}\right)$ and $\operatorname{ker}\left(\rho_{\left(v, e_{v}\right)}\right)$. Finally, the principal part of the locally filled section is the sc-smooth map

$$
\overline{\mathbf{f}}: \widehat{O} \rightarrow F:(v, e) \mapsto \mathbf{f}(v, e)+\mathbf{f}^{c}(v, e) .
$$

Since $f(0,0)=0$, the linearization of the section $f$ of the bundle $K^{\mathcal{R}} \rightarrow O$ at the point $(0,0) \in O$,

$$
\left.f^{\prime}(0,0)\right): T_{(0,0)} O \rightarrow \operatorname{ker}\left(1-\rho_{(0,0)}\right)=p^{-1}(0,0),
$$

is equal to $D \mathbf{f}(0,0) \mid T_{(0,0)} O$. We have to compare it to the linearization $D \overline{\mathbf{f}}(0,0): W \oplus$ $E \rightarrow F$. Here $W$ is the sc-Banach space containing the relatively open neighborhood $V$ of 0 in a partial quadrant $C \subset W$. According to the splitting $E=\pi_{0}(E) \oplus\left(1-\pi_{0}\right)(E)$ we shall split $\delta e \in E$ into $\delta e=(\delta a, \delta b)$ so that the tangent space at the point $(v, e)=$ $(0,0) \in O$ becomes $T_{(0,0)} O=\left\{(\delta w, \delta e) \in W \oplus E \mid \pi_{0}(\delta e)=\delta e\right\}=\{(\delta w, \delta a) \in$ $\left.W \oplus \pi_{0}(E)\right\}$ and compute

$$
\begin{aligned}
& D \overline{\mathbf{f}}(0,0)(\delta w, \delta e) \\
& \quad=D \mathbf{f}(0,0)(\delta w, \delta e)+D \mathbf{f}^{c}(0,0)(\delta w, \delta e) \\
& =D \mathbf{f}(0,0)(\delta w, \delta a)+D \mathbf{f}(0,0)(0, \delta b)+D \mathbf{f}^{c}(0,0)(\delta w, \delta a)+D \mathbf{f}^{c}(0,0)(0, \delta b) \\
& =D \mathbf{f}(0,0)(\delta w, \delta a)+D \mathbf{f}^{c}(0,0)(\delta w, \delta a)+D \mathbf{f}^{c}(0,0)(0, \delta b) \\
& =: f^{\prime}(0,0)(\delta w, \delta a)+B(\delta w, \delta a)+C(\delta b) .
\end{aligned}
$$

We have concluded from the identity $\mathbf{f}(v, e)=\mathbf{f}\left(v, \pi_{v}(e)\right)$ that

$$
D \mathbf{f}(0,0)(0, \delta b)=0 \text {. }
$$

In addition, since for fixed $v$ the map $\mathbf{f}^{c}\left(v, \pi_{v}(e)+\left(1-\pi_{v}\right)(e)\right)$ is linear in $\left(1-\pi_{v}\right)(e)$, we conclude that $D \mathbf{f}^{c}(0,0)(0, \delta b)=\mathbf{f}^{c}(0, \delta b)$. Since $(0,0) \in O$ is a smooth point, the map

$$
C: \operatorname{ker}\left(\pi_{0}\right) \rightarrow \operatorname{ker}\left(\rho_{(0,0)}\right): \delta b \mapsto \mathbf{f}^{c}(0, \delta b)
$$

is a linear sc-isomorphism, by the definition of a filler. The sc-operator $W \oplus \operatorname{ker}\left(\pi_{0}\right) \rightarrow$ $\operatorname{ker}\left(\rho_{(0,0)}\right)$ defined by $(\delta w, \delta a) \rightarrow D \mathbf{f}^{c}(0,0)(\delta w, \delta a)$ vanishes.

Hence our total linear sc-operator $D \overline{\mathbf{f}}(0,0)$ has the matrix form

$$
\left[\begin{array}{c}
(\delta w, \delta a) \\
\delta b
\end{array}\right] \mapsto\left[\begin{array}{cc}
f^{\prime}(0,0) & 0 \\
0 & C
\end{array}\right] \cdot\left[\begin{array}{c}
(\delta w, \delta a) \\
\delta b
\end{array}\right],
$$

where $C$ is an sc-isomorphism. An sc-operator of this form is sc-Fredholm if and only if the linearization $f^{\prime}(0,0)$ is sc-Fredholm. In that case the Fredholm indices are the same. This completes the proof.

What we discussed in this paper is a minimal set of concepts needed to develop a Fredholm theory. The next paper will contain a treatment of implicit function theorems in the splicing context.

We refer the reader to the upcoming volume [6] for a more exhaustive list of splicing constructions and constructs. 
Acknowledgments. We would like to thank P. Albers and U. Hryniewicz for helpful comments.

Research of H. Hofer was partially supported by NSF grant DMS-0603957; that of K. Wysocki by NSF grant DMS-0606588; and that of E. Zehnder by TH-project.

\section{References}

[1] Bourgeois, F., Eliashberg, Y., Hofer, H., Wysocki, K., Zehnder, E.: Compactness results in symplectic field theory. Geom. Topol. 7, 799-888 (2003) Zbl pre02062477 MR 2026549

[2] Eliashberg, Y., Givental, A., Hofer, H.: Introduction to symplectic field theory. Geom. Funct. Anal. 2000, Special Volume, Part II, 560-673 Zbl 0989.81114 MR 1826267

[3] Eliasson, H.: Geometry of manifolds of maps. J. Differential Geom. 1, 169-194 (1967) Zbl 0163.43901 MR 0226681

[4] Gromov, M.: Pseudoholomorphic curves in symplectic geometry. Invent. Math. 82, 307-347 (1985) Zbl 0592.53025 MR 0809718

[5] Hofer, H.: A general Fredholm theory and applications. In: Current Developments in Mathematics, D. Jerison et al. (eds.), International Press (2006)

[6] Hofer, H., Wysocki, K., Zehnder, E.: Fredholm Theory in Polyfolds I: Functional Analytic Methods. Book in preparation

[7] Hofer, H., Wysocki, K., Zehnder, E.: Fredholm Theory in Polyfolds II: The Polyfolds of Symplectic Field Theory. Book in preparation

[8] Hofer, H., Wysocki, K., Zehnder, E.: A general Fredholm theory II: Implicit function theorems. Preprint

[9] Hofer, H., Wysocki, K., Zehnder, E.: A general Fredholm theory III: Fredholm functors and polyfolds. Paper in preparation

[10] Hofer, H., Wysocki, K., Zehnder, E.: A general Fredholm theory IV: Operations. Paper in preparation

[11] Hofer, H., Wysocki, K., Zehnder, E.: Applications of polyfold theory I: Gromov-Witten theory. Paper in preparation

[12] Hofer, H., Wysocki, K., Zehnder, E.: Applications of polyfold theory II: The polyfolds of symplectic field theory. Paper in preparation

[13] Jänich, K.: On the classification of $O(n)$-manifolds. Math. Ann. 176, 53-76 (1968) Zbl 0153.53801 MR 0226674

[14] Lang, S.: Fundamentals of Differential Geometry. Grad. Texts in Math. 191, Springer (1999) Zbl 0932.53001 MR 1666820

[15] Laures, G.: On cobordism of manifolds with corners. Trans. Amer. Math. Soc. 352, 56675688 (2000) Zbl 0954.55008 MR 1781277

[16] McDuff, D.: Groupoids, branched manifolds and multisections. J. Symplectic Geom. 4, 259315 (2006)

[17] Moerdijk, I.: Orbifolds as groupoids: An introduction. In: Orbifolds in Mathematics and Physics (Madison, WI, 2001), Contemp. Math. 310, Amer. Math. Soc., 205-222 (2002) Zbl 1041.58009 MR 1950948

[18] Moerdijk, I., Mrčun, J.: Introduction to Foliations and Lie Groupoids. Cambridge Stud. Adv. Math. 91, Cambridge Univ. Press (2003) Zbl 1029.58012 MR 2012261

[19] Triebel, H.: Interpolation Theory, Function Spaces, Differential Operators, North-Holland, Amsterdam (1978) Zbl 0387.46033 MR 0500580 